BMC

Genomics

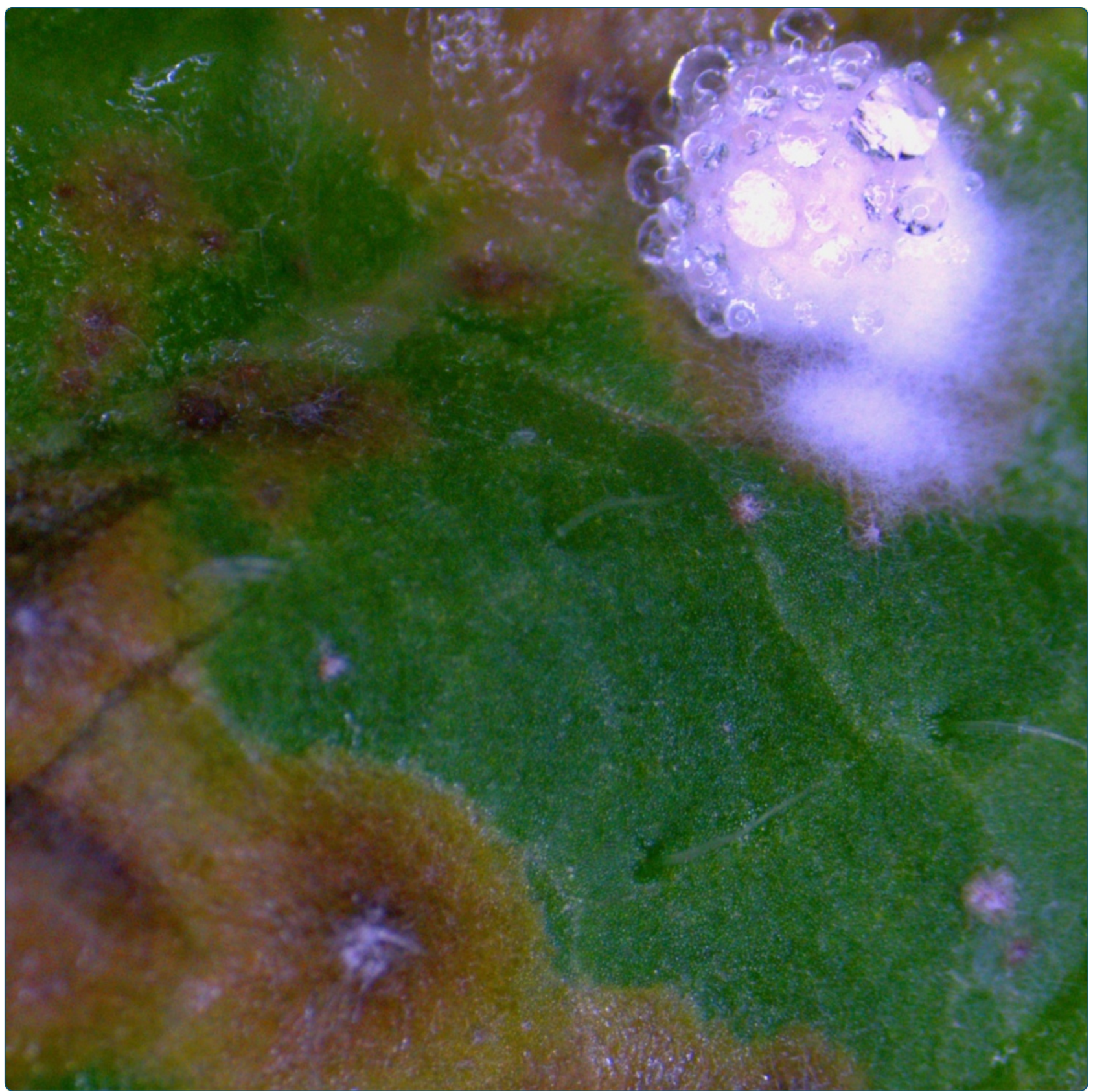

Secretome analysis reveals effector candidates associated with broad host range necrotrophy in the fungal plant pathogen Sclerotinia sclerotiorum

Guyon et al. 


\title{
Secretome analysis reveals effector candidates associated with broad host range necrotrophy in the fungal plant pathogen Sclerotinia sclerotiorum
}

\author{
Koanna Guyon ${ }^{1,2}$, Claudine Balagué ${ }^{1,2}$, Dominique Roby ${ }^{1,2}$ and Sylvain Raffaele ${ }^{1,2^{*}}$
}

\begin{abstract}
Background: The white mold fungus Sclerotinia sclerotiorum is a devastating necrotrophic plant pathogen with a remarkably broad host range. The interaction of necrotrophs with their hosts is more complex than initially thought, and still poorly understood.

Results: We combined bioinformatics approaches to determine the repertoire of S. sclerotiorum effector candidates and conducted detailed sequence and expression analyses on selected candidates. We identified 486 S. sclerotiorum secreted protein genes expressed in planta, many of which have no predicted enzymatic activity and may be involved in the interaction between the fungus and its hosts. We focused on those showing (i) protein domains and motifs found in known fungal effectors, (ii) signatures of positive selection, (iii) recent gene duplication, or (iv) being S. sclerotiorum-specific. We identified 78 effector candidates based on these properties. We analyzed the expression pattern of 16 representative effector candidate genes on four host plants and revealed diverse expression patterns.
\end{abstract}

Conclusions: These results reveal diverse predicted functions and expression patterns in the repertoire of S. sclerotiorum effector candidates. They will facilitate the functional analysis of fungal pathogenicity determinants and should prove useful in the search for plant quantitative disease resistance components active against the white mold.

Keywords: Sclerotinia sclerotiorum. Effectors, Gene expression, Secretome, Necrotrophic fungal, Pathogen, Arabidopsis thaliana

\section{Background}

The white mold fungus Sclerotinia sclerotiorum (Lib.) de Bary is a cosmopolitan necrotrophic pathogen infecting over 400 plant species. It is among the most devastating pathogens of soybean, rapeseed and sunflower, causing several hundred million dollar losses annually at the preand postharvest stages [1]. S. sclerotiorum host range is remarkably broad, with fruit and vegetable productions also being severely impacted [2]. S. sclerotiorum and its close relative the grey mould fungus Botrytis cinerea are among the few fungal pathogens considered as typical necrotrophs. As such, they derive energy to complete

\footnotetext{
* Correspondence: Sylvain.raffaele@toulouse.inra.fr

'INRA, Laboratoire des Interactions Plantes-Microorganismes (LIPM), UMR441, F-31326, Castanet-Tolosan, France

${ }^{2}$ CNRS, Laboratoire des Interactions Plantes-Microorganismes (LIPM), UMR2594, F-31326, Castanet-Tolosan, France
}

their life cycle mostly from dead plant cells, as opposed to biotrophs that feed on living plant cells.

There is now ample evidence that biotrophic and hemibiotrophic fungi secrete specialized effector proteins manipulating host cell physiology to obtain nutrients, suppress plant defense and ultimately promote infection [3]. Effectors may also trigger plant defense responses, leading to plant resistance, when recognized directly or indirectly by the plant immune system in a gene-for-gene relationship. This results from a co-evolutionary arms race between pathogen effectors, their plant targets, and components of the plant immune system [4]. Necrotrophs have long been considered as less adapted, secreting mostly degrading enzymes and toxins that unspecifically trigger programmed cell death (PCD) and dismantle plant cells. 
However, host specific necrotrophs such as Cochliobolus victoriae secrete effector proteins translocated into plant cells that interact with specific corresponding host proteins to facilitate disease progression [5,6]. This involves the activation of plant PCD instead of its suppression as in the case of infection by biotrophic pathogens. S. sclerotiorum also produces the non-proteic pathogenicity determinant oxalic acid. This molecule induces the synthesis of reactive oxygen species (ROS) and triggers plant PCD late during infection, but has the opposite effect, suppressing ROS burst and PCD induction, at the early stages of infection [7]. The SSITL secreted integrin-like protein of S. sclerotiorum promotes virulence and delays the activation of plant defense responses, supporting the view that S. sclerotiorum secretes effectors to finely manipulate plant physiology [8]. In addition, enzymes secreted by necrotrophs can act as virulence factors independently of their catalytic activity [9]. Effector repertoires vary considerably, notably according to pathogens lifestyle [10], and it is becoming clear that interactions between necrotrophs and their host plants are considerably more complex and subtle than previously considered. What is the effector candidate repertoire associated with broad host range necrotrophy remains unclear. As a first step towards elucidating the molecular bases for colonization by S. sclerotiorum, its repertoire of effector candidates needs to be determined.

The recent release of genome sequences for a number of plant pathogenic fungi facilitated the search for effector candidates (ECs) at the genome level [11]. Nevertheless, considering that pathogen effector repertoires are typically lineage-specific, the identification of effectors remains challenging [4]. The analysis of S. sclerotiorum genome sequence uncovered sets of genes associated with the manipulation of redox status, including enzymes of OA biosynthesis, the degradation of plant cell wall, and 603 secreted proteins with other functions [12]. Known hallmarks of fungal effectors such as the presence of signal peptides and absence of transmembrane domains, small size and amino-acid composition generally produce lists of hundreds of potential effectors in a given pathogen. Therefore, more sophisticated approaches are required to pinpoint the most relevant ECs for the promotion of infection in S. sclerotiorum secretome.

A limited number of known fungal effector families show conservation at the sequence level or similar predicted functions. This is notably the case for the toxin and cell death elicitor proteins of the Necrosis and ethylene-inducing Like Proteins (NLPs), the ceratoplatanin, cyanovirin-N homology $(\mathrm{CVNH})$ and ECP2 families [13-16]. The growing number of characterized fungal effectors suggests conservation at the biochemical function level in the overall effector repertoire of several fungal pathogens. The ability to bind chitin or other cell wall oligosaccharides, masking the presence of the pathogen or dampening damage-induced plant responses, is a feature common to effectors from multiple fungal pathogens [17-20]. Fungal effectors harboring a protease inhibitor activity are also common [21-24]. The biochemical activity of a few other fungal effectors such as $M$. oryzae Fungalysin metalloprotease AvrPita [25], $U$. maydis chorismate mutase cmu1 [26] and peroxidase inhibitor PEP1 [27] may also be part of the arsenal of effector functions in multiple fungal lineages. This hypothesis suggests that thorough annotation of protein domains and prediction of biochemical function of secreted proteins may prove useful to identify novel effectors in S. sclerotiorum.

However, a majority of effectors do not show significant similarity to known sequences in other organisms nor obvious protein domains. Yet other genomic characteristics may help identify EC genes. The rapid evolution of effector genes allows the fungi to overcome selection pressures induced by resistant plant cultivars. A high ratio of non-synonymous over synonymous substitutions $(\mathrm{Ka} / \mathrm{Ks})$ in alleles from related strains is a frequently used proxy for inferring fast gene evolution and the action of positive selection [28]. This approach has been used to reveal ECs in several filamentous plant pathogen lineages [29-34]. Positive selection has been detected in B. cinerea genome [35] suggesting that it may be used to mine $S$. sclerotiorum genome for ECs. Second, gene duplication is another hallmark of several known fungal effector genes, such as the ToxB host specific toxin of Pyrenophora tritici-repentis [36,37]. Third, genomic regions with high repeat and transposable element content are enriched in effector genes in several lineages of plant pathogens [37-39] suggesting that genome architecture analysis can assist in the search for EC genes. Finally, effectors can alter host cell function by mimicking plant peptides [40]. These ECs likely elude functional annotation on the basis of primary amino-acid sequence, but may be revealed using three-dimensional structure prediction.

As opposed to Oomycete pathogen genomes in which many effector genes can be identified through conserved sequence motifs $[29,41]$, the use of conserved sequence motifs, such as the $\mathrm{Y} / \mathrm{F} / \mathrm{WxC}$ motif [42], has proven limited in revealing fungal effectors across lineages. However, the presence of a signal peptide directing protein secretion and gene expression in planta are relatively universal properties of effectors that can be exploited as first filters to narrow down the list of effector candidates in fungal genomes.

S. sclerotiorum effector proteins would be useful as probes to search for resistance components in plants and to design strategies for inhibiting infections by this devastating but poorly characterized pathogen. In this study, we report a diverse repertoire of S. sclerotiorum effector candidates revealed by an in depth analysis of its predicted secretome. We combined refined secretome 
annotation, phylogeny, selection and gene duplication analyses, and three-dimensional structure prediction to identify 78 ECs. Among those, we highlight a predicted subtilisin inhibitor, a xylanase, a duplicated gene of unknown function and three toxin mimics as high priority candidates for functional studies. We analyzed in planta expression pattern for 16 EC genes and revealed hostblind and host-regulated ECs.

\section{Results}

\section{Definition and annotation of S. sclerotiorum secretome}

In the original analysis of $S$. sclerotiorum genome, secreted proteins were predicted using SignalP, TargetP and TMHMM, and annotated using Interproscan [12]. To identify and explore candidate effectors in the genome of S. sclerotiorum, we built a bioinformatics workflow exploiting genomic features typical of known filamentous plant pathogen effectors. We first refined the prediction of secreted proteins combining predictions from SignalP2 and 4 to identify 1070 proteins with a secretion signal. To evaluate the sensitivity of this prediction, we applied the same methodology on a list of 1985 eukaryotic secreted proteins validated experimentally from the SPdb [43]. We retrieved 1971 proteins predicted as secreted, corresponding to a true positive rate of $99.34 \%$. Next, we removed 172 proteins predicted by TMHMM to harbor transmembrane helices, then 153 proteins predicted by GPIsom to harbor glycophosphatidylinositol anchor motifs, that likely represent surface proteins rather than secreted effectors. This resulted in a list of 745 predicted secreted proteins. To increase the likelihood of identifying genes relevant for infection, we selected those for which there is evidence for expression in planta based on publicly available EST and microarrays data. We considered genes expressed, even if not induced, during interaction with rapeseed, sunflower or tomato (see Methods). Although this filtering pipeline likely excluded relevant effector candidates, it resulted in a total of 486 genes encoding predicted secreted proteins expressed in planta (SPEPs, Figure 1a, Additional file 1: Table S1).

Next, we performed three different effector-oriented analyses on the 486 SPEP genes. First, we used Blast2GO, PFAM domain and nuclear localization signal (NLS) searches to annotate 326 SPEPs (Figure 1b). We built a database of known fungal effectors and explored the literature to select $31 \mathrm{~S}$. sclerotiorum ECs among annotated SPEP genes. Second, we defined clusters of orthologous genes (COGs) between S. sclerotiorum and B. cinerea predicted genes using Inparanoid (Figure 1c). A total of 197 SPEP genes grouped in COGs. We aligned S. sclerotiorum and B. cinerea orthologs for these 197 gene pairs and calculated ratios of non synonymous over synonymous substitutions $(\mathrm{Ka} / \mathrm{Ks})$ to identify five ECs with signature of positive selection. The 289 SPEP genes with no ortholog

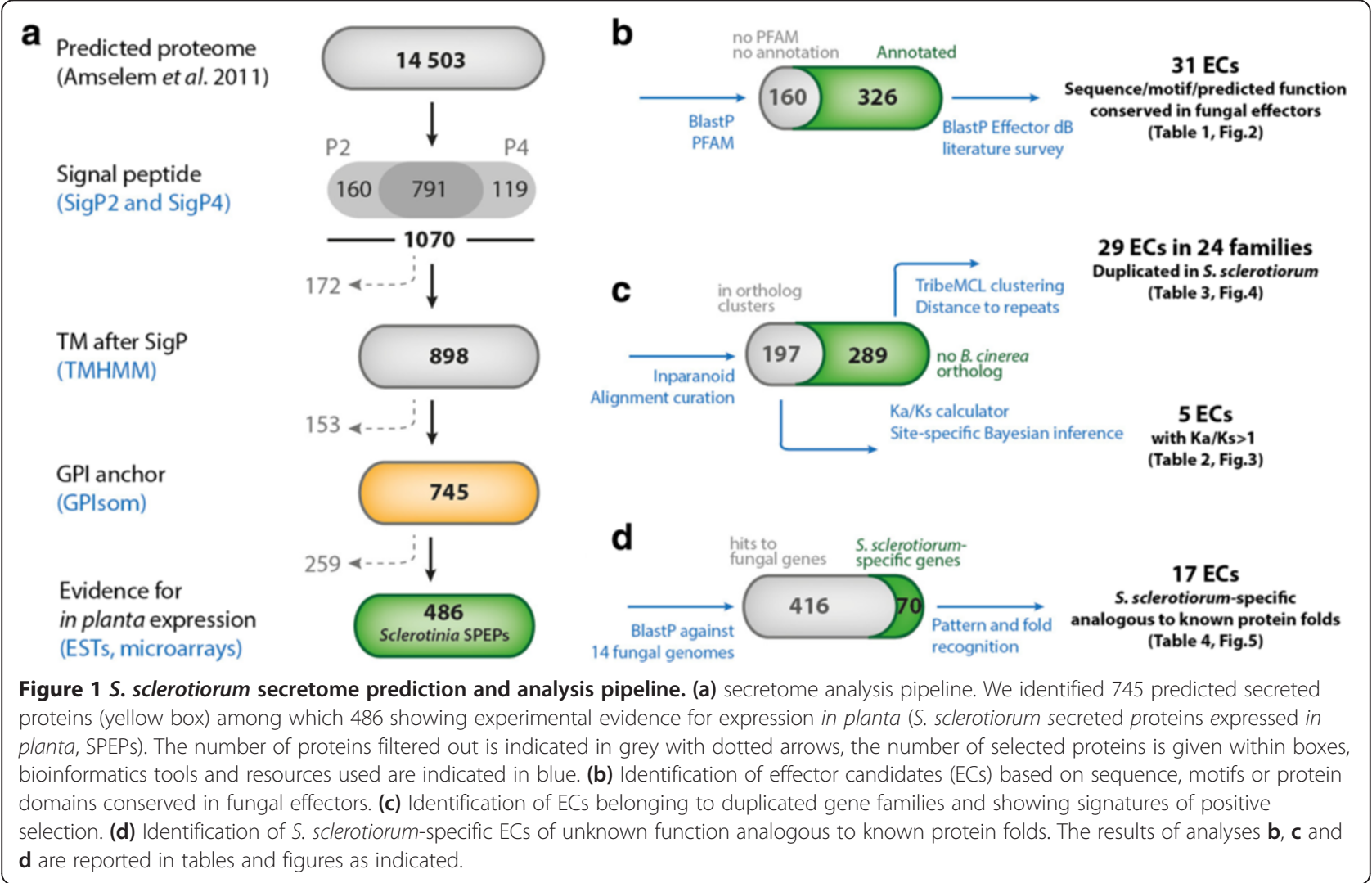


in $B$. cinerea were grouped into clusters based on sequence similarity to identify 29 ECs distributed in 24 families containing genes duplicated in S. sclerotiorum. Finally, we analyzed the taxonomic distribution of SPEP genes across the kingdom Fungi using BlastP searches against a database of 14 complete genomes representative of all major fungal lineages. This identified $70 \mathrm{~S}$. sclerotiorum-specific SPEP genes, most of which had no annotation (Figure 1d). We used protein structure prediction and pattern and fold recognition searches to identify 17 ECs analogous to known protein fold encoded by $S$. sclerotiorum-specific SPEP genes.

Using effector-oriented analyses, we identified four lists of ECs, containing a total of 78 EC genes (four being common to two lists). We could not predict any enzymatic activity encoded by $33 \%$ of the SPEP genes (160), suggesting that $S$. sclerotiorum effector repertoire encodes diverse functions that are not restricted to plant cell degrading enzymes. Besides, 59.5\% of the SPEP genes (289) did not cluster in $B$. cinerea COGs, revealing a relatively high degree of divergence from this closely related fungal pathogen.

\section{Sclerotinia effector candidates showing conserved domains}

Some fungal effectors show conserved protein domains and biochemical functions that can be identified at the protein sequence level. In a first approach to identify ECs, we used (i) PFAM annotations, (ii) nuclear localization signal (NLS) predictions, and (iii) BlastP searches against known fungal effectors. We found 326 annotated SPEP genes among which we selected 13 as effector candidates based on the presence of specific PFAM domains (Table 1). Indeed, six predicted SPEPs (SS1G_00642, SS1G_02014, SS1G_04786, SS1G_09392, SS1G_12336, SS1G_14184) included chitin-recognition or chitin-binding domains (PF00187, PF03067) and one SPEP (SS1G_12509) contained LysM domains (PF01476). SS1G_07836 was annotated as a concanavalin A-like lectin/glucanase that binds to complex carbohydrates, and harbors a Peptidase A4 (PF01828) with a typical lectin fold. SS1G_08698 contains a Ricin type beta-trefoil lectin domain (PF00652) typical of ricin-like toxins. These predicted SPEPs are relevant ECs considering that chitin- and carbohydrate-binding activity has been reported for several fungal effector families. We also selected three predicted SPEPs (SS1G_01593, SS1G_03282, SS1G_12605) with protease inhibitor domains (PF05922), another activity found in known fungal effectors. We identified two predicted SPEPs (SS1G_ 03611 and SS1G_13935) with a CFEM cystein-rich fungal effector motif (PF05730). Second, we selected another 11 SPEP genes based on the presence of at least one predicted NLS in their sequence (Table 1). The co-occurrence of a secretion signal and a NLS in encoded proteins suggest that they may be active in the plant nucleus.
Consistent with a nuclear activity, SS1G_01866 and SS1G_05895 also harbored transcription factor domains. Third, we selected 7 predicted SPEPs that were retrieved based on sequence similarity to known effectors or ECs from other plant pathogenic fungi (Table 1). We recovered the two necrosis and ethylene inducing peptides SsNEP1 and SsNEP2 (SS1G_03080 and SS1G_11912) described in [44] sharing 40\% identity with various NEP-like proteins, and harboring a characteristic PFAM domain (PF05630). We selected SS1G_00849 for sharing 59\% identity with Colletotrichum hingginsianum effector candidate 91 (CHEC91, [45]). There was no protein domain identified in this protein but three-dimensional structure prediction indicates that it is analogous to Alternaria alternata AltA-1 allergen. We selected SS1G_02904 for sharing $35 \%$ identity with $C$. hingginsianum effector candidate 80 (CHEC80, [45]). It features a Cyanovirin- $\mathrm{N}$ homology domain (PF13639) that corresponds to a carbohydratebinding module $[15,46]$. We identified SS1G_08858 showing 25\% identity with $M$. oryzae effector AvrPita (MGG_15730, [25]) and harboring a M35 metalloprotease domain (PF02102). Finally we selected SS1G_10096 as similar ( $>60 \%$ identity) to pathogen-associated molecular patterns (PAMP) with a cerato-platanin domain (PF07249) $[14,45,47,48]$. These 31 ECs identified based on protein annotations showed that the activity of S. sclerotiorum secretome may not be limited to typical cell death elicitors but is predicted to cover a wide range of functions known for fungal effectors, including chitin binding, proteases and protease inhibitors.

\section{A family of subtilisin-inhibitor effector candidates conserved in Ascomycetes}

Effectors with a protease inhibitor activity have been described in fungal plant pathogens with a biotrophic phase of infection $[22,49]$. We identified three S. sclerotiorum candidate effectors (SS1G_01593, SS1G_03282 and SS1G_ 12605 , Table 1) with a serine protease inhibitor I9 domain (PF05922) that corresponds to the propeptide inhibitor domain of subtilisins. To document the taxonomic distribution of SS1G_01593 homologs in fungi, we performed a BlastP search against the predicted proteome of 234 fungal species. Using signal peptide predictions, we identified 99 secreted homologs across 97 species. Secreted homologs of SS1G_01593 are restricted to Ascomycetes and found in all Leotiomycete species considered (Figure 2a). At the sequence level, homologs of SS1G_01593 are clearly distinct from C. fulvum Avr2, U. maydis Pit2, Uromyces fabae RTP1p and M. lini AvrP123, suggesting that SS1G_01593 family represent a distinct class of protease inhibitor effectors. To get insights into SS1G_01593 function and evolution, we predicted the 3D structure of the protein and mapped residue conservation among the 99 secreted homologs on this structure. SS1G_01593 
Table 1 List of $31 \mathrm{~S}$. sclerotiorum effector candidates selected based on their annotation SPEPs containing PFAM domains found in fungal effectors

\begin{tabular}{lllll}
\hline Protein ID & Annotation & PFAM domain(s) & Length & Comments \\
\hline SS1G_00642 & Chitin binding protein & Chitin recognition protein (PF00187.14) & 563 & 426 \\
SS1G_02014 & Chitin binding protein & Chitin binding domain (PF03067) & 399 \\
SS1G_04786 & Chitin binding protein & Chitin recognition protein (PF00187.14) & 398 \\
SS1G_09392 & Starch binding domain & Chitin binding domain (PF03067) & \\
& containing protein & & 294 \\
SS1G_12336 & Chitin binding protein & Chitin binding domain (PF03067) & 245 \\
SS1G_14184 & Agglutinin isolectin 3-like & Chitin recognition protein (PF00187.14) & 447 \\
SS1G_12509 & LysM domain protein & LysM (PF01476) & 252 \\
SS1G_07836 & Concanavalin A lectin glucanase & Peptidase_A4 (PF01828) & 409 \\
SS1G_08698 & Ricin-type toxin & Ricin-type beta-trefoil lectin domain (PF00652) & 95 \\
SS1G_01593 & Serine protease inhibitor & Peptidase inhibitor 19 (PF05922) & 522 \\
SS1G_03282 & Serine protease inhibitor & Peptidase inhibitor 19 (PF05922) & 400 \\
SS1G_12605 & Alkaline serine protease alp1 & Peptidase inhibitor 19 (PF05922) & 119 \\
SS1G_03611 & Cystein-rich protein & CFEM (PF05730) & 529 \\
SS1G_13935 & Cystein-rich protein & CFEM (PF05730) &
\end{tabular}

SPEPs containing nuclear localization signal (NLS)

\begin{tabular}{|c|c|c|c|c|}
\hline Protein ID & Annotation & PFAM domain(s) & Length & Comments \\
\hline SS1G_01866 & Ring-7 protein & $\begin{array}{l}\text { PHD-like zinc-binding domain (PF13771); } \\
\text { Ring finger domain (PF13639) }\end{array}$ & 425 & NLS (118-141) \\
\hline SS1G_03146 & - & - & 193 & NLS (147-155) \\
\hline SS1G_04309 & - & - & 177 & NLS (82-87) \\
\hline SS1G_05895 & $\begin{array}{l}\text { Zinc finger CCCH-type domain } \\
\text { containing protein }\end{array}$ & Zinc finger C-x8-C-x5-C-x3-H type (PF00642) & 310 & NLS (256-291) \\
\hline SS1G_05938 & - & - & 212 & NLS (143-150) \\
\hline SS1G_06787 & - & - & 430 & NLS (189-250; 340-349) \\
\hline SS1G_06890 & - & - & 284 & NLS (66-120; 143-167; 225-245) \\
\hline SS1G_07404 & - & DUF3108 (PF11306) & 282 & NLS (65-79; 118-138; 223-233) \\
\hline SS1G_09050 & - & - & 454 & NLS (410-413) \\
\hline SS1G_11108 & Ribosomal protein s17 & - & 373 & NLS (338-348) \\
\hline SS1G_13142 & - & - & 131 & NLS (89-99) \\
\hline \multicolumn{5}{|c|}{ SPEPs showing homology to fungal effectors or fungal effector candidates } \\
\hline Protein ID & Annotation & PFAM domain(s) & Length & Comments \\
\hline SS1G_03080 & SsNEP1 & Necrosis inducing protein (PF05630) & 246 & $\begin{array}{l}\text { Homolog to B. cinerea NEP1 } \\
\text { (Staats et al., 2007); P. Sojae } \\
\text { NIP (Qutob et al., 2002); studied } \\
\text { in (Bashi et al., 2011) }\end{array}$ \\
\hline SS1G_11912 & SsNEP2 & Necrosis inducing protein (PF05630) & 245 & $\begin{array}{l}\text { Homolog to B. cinerea NEP1 } \\
\text { (Staats et al., 2007); P. Sojae } \\
\text { NIP (Qutob et al., 2002); studied } \\
\text { in (Bashi et al., 2011) }\end{array}$ \\
\hline SS1G_00849 & AltA-1 allergen analog & - & 152 & $\begin{array}{l}\text { Homolog to C. hingginsianum } \\
\text { HE651255_CHEC91 } \\
\text { Kleemann et al., 2012) }\end{array}$ \\
\hline SS1G_02904 & CVNH protein & CyanoVirin-N Homology domain (PF08881) & 169 & $\begin{array}{l}\text { Homolog to C. hingginsianum } \\
\text { HE651243_CHEC80 } \\
\text { Kleemann et al., 2012) }\end{array}$ \\
\hline SS1G_08858 & Deuterolysin metalloprotease & $\begin{array}{l}\text { Deuterolysin metalloprotease (M35) } \\
\text { family (PF02102) }\end{array}$ & 354 & $\begin{array}{l}\text { Homolog to M. oryzae AvrPita } \\
\text { (MGG_15370 - Orbach et al., 2000) }\end{array}$ \\
\hline
\end{tabular}


Table 1 List of $31 \mathrm{~S}$. sclerotiorum effector candidates selected based on their annotation (Continued)

\begin{tabular}{|c|c|c|c|}
\hline SS1G_10096 & Cerato-platanin (PF07249) & 137 & $\begin{array}{l}\text { Homolog to B. cinerea BcSpl1 } \\
\text { (Frias et al., 2011); H. atroviridis } \\
\text { EPL1 (Seidl et al., 2006); M. oryzae } \\
\text { MSP1 Jeong et al., 2007); } \\
\text { C. hinginsianum HE651160_CHEC5 } \\
\text { Kleemann et al., 2012) }\end{array}$ \\
\hline
\end{tabular}

Results from Blast2GO automated annotation, PFAM domain searches and BlastP against known fungal effectors were considered. Table entries are ordered as in main text. SPEP, S. sclerotiorum secreted protein induced in planta.

protein is predicted to adopt the structure of subtilisin pro-domain consisting of 4 beta-sheets facing two alphahelices (Figure 2b). The beta-sheets, interacting with the catalytic domain in subtilisins, are well conserved in fungi, whereas the exposed alpha-helices are more variable, suggesting that the ability to interact with subtilisins is conserved.

\section{Sclerotinia effector candidates showing high $\mathrm{Ka} / \mathrm{Ks}$ ratios} According to the red queen hypothesis, fungal pathogen effectors are predicted to evolve rapidly. Signatures of natural selection were used to identify effectors and elicitors of immune responses in bacterial, oomycete and fungal plant pathogens $[29,35,50,51]$. To detect signatures of natural selection in SPEP genes, we calculated $\mathrm{Ka} / \mathrm{Ks}$ ratios using Yn00 [28] on alignments between S. sclerotiorum and B. cinerea 197 core ortholog pairs. We obtained $\mathrm{Ka} / \mathrm{Ks}$ values ranging from 0.009 to 6 , with a median value of $\sim 0.1$. Notably 5 genes $(2.5 \%)$ showed $\mathrm{Ka} / \mathrm{Ks}>1$, suggesting positive selection (Figure 3a, Table 2). To identify codon sites under positive selection in the five SPEP genes showing $\mathrm{Ka} / \mathrm{Ks}>1$, we used a Bayesian inference approach on alignments of S. sclerotiorum 1980, B. cinerea B05.10 and B.cinerea t4 orthologs. We could not detect sites with $\mathrm{Ka} / \mathrm{Ks}>1$ in SS1G_04551 and SS1G_07158 using this dataset. We detected 7, 50 and 20 sites with $\mathrm{Ka} / \mathrm{Ks}>1$ in SS1G_07749, SS1G_10165 and SS1G_10617 mature proteins respectively. The pvalue for positive selection using M8 and M8e models was 8.2 $\mathrm{e}^{-2}, 3.8 \mathrm{e}^{-4}$ and $3.0 \mathrm{e}^{-1}$ for SS1G_07749, SS1G_10165 and SS1G_10617 respectively (Table 2). Interestingly, SS1G_07749 encodes a putative xylanase that may function as a virulence factor such as xylanases from other fungi $[9,52]$. To get insights on the constraints shaping the evolution of SS1G_07749 xylanase, we predicted the 3D structure of SS1G_07749 mature protein and mapped the local $\mathrm{Ka} / \mathrm{Ks}$ ratios on this structure (Figure $3 \mathrm{~b}$ ). All seven selected sites correspond to surface-exposed residues with three residing at the surface potentially interacting with plant xylanase inhibitors (R36, D74, Q76), three at the surface of the region corresponding to B. cinerea Xyn11 necrotizing peptide (N174, L189, S200) and one (L43) modifying the surface of the substrate-binding pocket. These findings are consistent with adaptation to host and the possible involvement of SS1G_07749 in virulence.

\section{Sclerotinia effector candidates encoded by recently duplicated genes}

Several effectors of filamentous plant pathogens evolved through gene duplication followed by rapid diversification
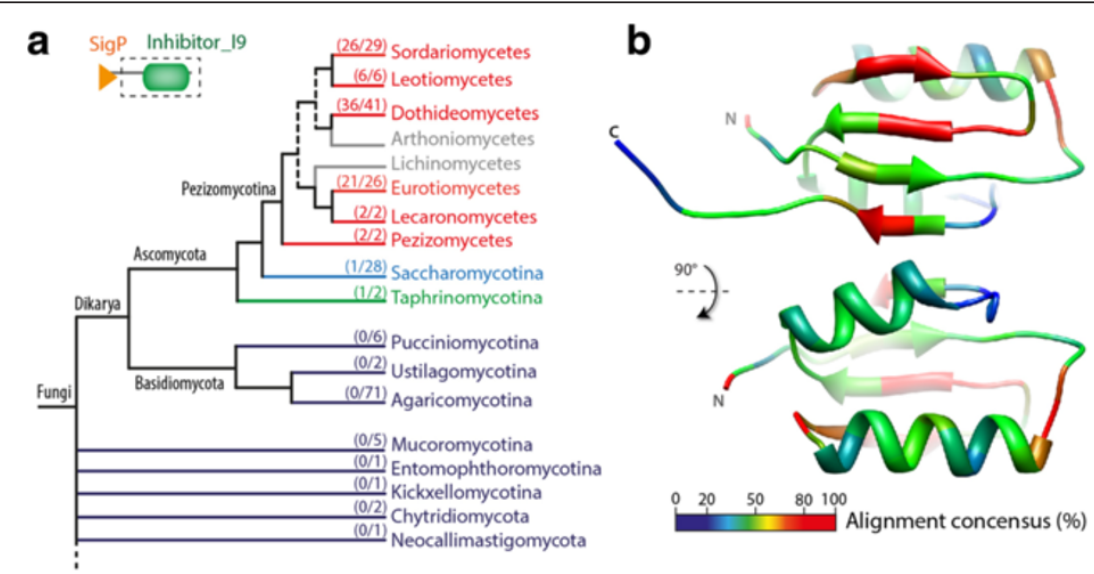

Figure 2 Sclerotinia effector candidates showing conserved domains: example of a novel class of protease inhibitors. (a) The domain organization of SS1G_01593, a S. sclerotiorum effector candidate with a peptidase inhibitor 19 domain (PF05922), and the distribution of its homologs across fungal taxonomy shown on a tree assembled based on published phylogenies, with branches color-coded from blue to red based on the percentage of species in a given order harboring homologs. (b) The predicted 3D structure of SS1G_01593 with residues color-coded based on conservation in fungi. 

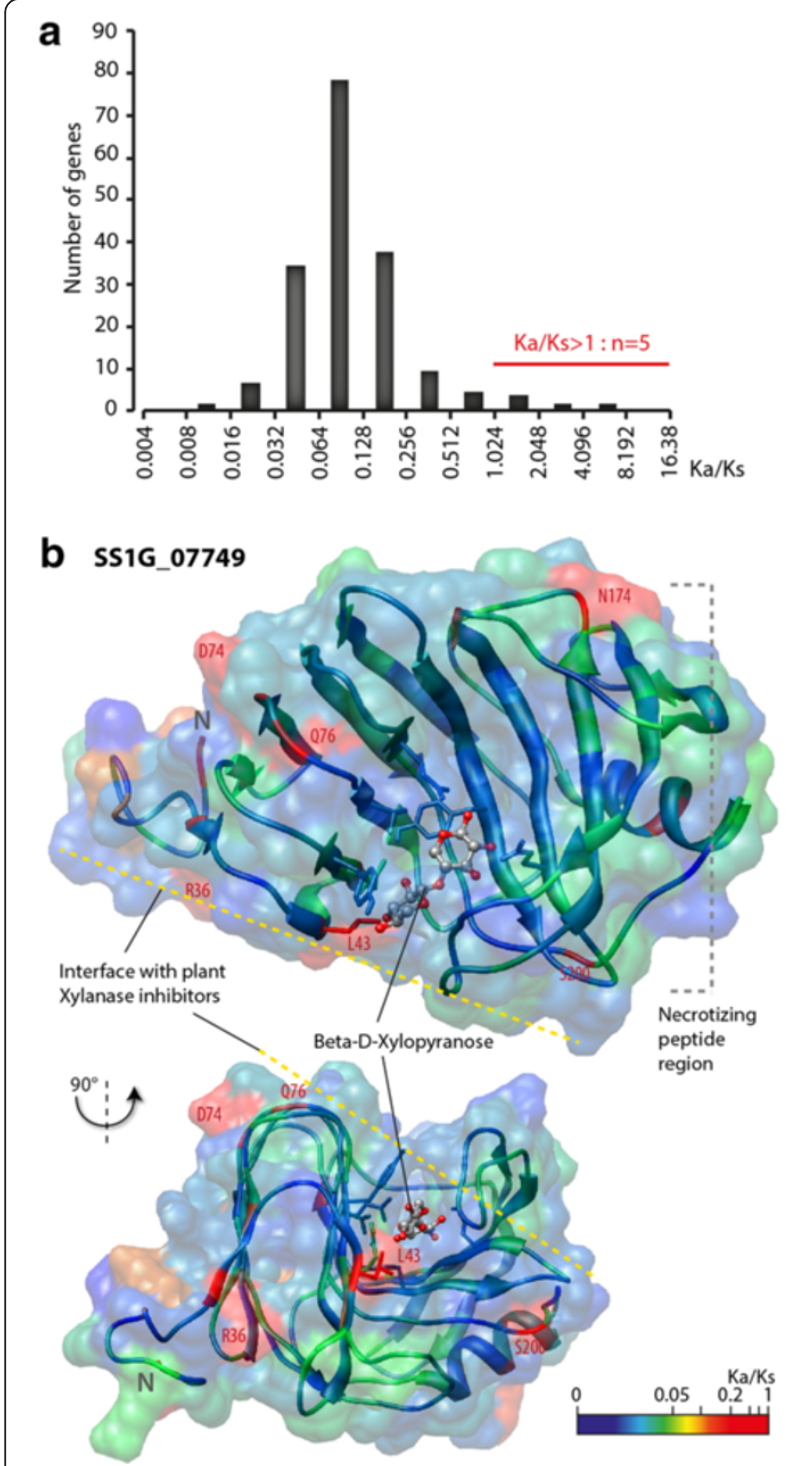

Figure 3 Sclerotinia effector candidates selected based on $\mathrm{Ka} / \mathrm{Ks}$ ratio. (a) Distribution of Ka/Ks ratio for the 197 SPEP genes with orthologs in B. cinerea, calculated with Yn00 method on pairwise ortholog alignments. (b) Predicted 3D protein structure of SS1G_07749, a member of the glycoside hydrolase 11 xylanase family with global $\mathrm{Ka} / \mathrm{Ks}=2$ in comparisons with $B$. cinerea orthologs. Residues of the 3D model are color-coded according to site-specific $\mathrm{Ka} / \mathrm{Ks}$ ratios calculated using Bayesian inference with M8 model [28]. Residues with $\mathrm{Ka} / \mathrm{Ks}>1$ are labeled on the structure. A putative Beta-D-Xylopyranose substrate molecule present in the 3b5I_B model, best structural analog of SS1G_07749, is shown as balls and sticks. The side chains of residues forming the predicted substrate binding site predicted by COFACTOR are show as sticks. The interface with plant xylanase inhibitors shown as a yellow dotted line was inferred from $[53,54]$ and the necrotizing peptide region shown as a grey dotted line was inferred from [9].

$[16,51]$. To identify SPEP genes that underwent duplications since the divergence of $S$. sclerotiorum with $B$. cinerea, we grouped proteins from their whole predicted proteomes based on sequence similarity using Markov clustering, and selected groups matching all three criterions: (i) containing at least 3 proteins, (ii) containing at least one S. sclerotiorum SPEP gene and (iii) containing more than $50 \%$ of proteins from S. sclerotiorum. We obtained 24 such groups including a total of $71 \mathrm{~S}$. sclerotiorum proteins among which $29 \mathrm{~S}$. sclerotiorum SPEP genes (Figure 4a, Table 3). Groups 051, 170, 175 and 204 contained only secreted proteins and probably carry effector-specific functions. Group 182 and 167 contained SPEP genes identified as ECs based on the presence of CFEM and LysM domains respectively (Table 1). SPEP genes from group 51, 80 and 204 had no conserved domain or homology to proteins of known function. In some pathogen genomes, the expansion of effector gene families is associated with the proximity to transposable elements [4]. To determine the repeat environment of S. sclerotiorum ECs, we annotated repeats in S. sclerotiorum genome, and calculated for all genes the distance to the nearest repeat. Median distance to repeats is 7.89 Kbp across S. sclerotiorum genome and $3.56 \mathrm{Kbp}$ for the 29 ECs that underwent recent duplications. Nine duplicated ECs are located less than $2 \mathrm{Kbp}$ apart from a retrotransposon and may have undergone duplication due to transposable element activity (Figure 4b, Table 3 ).

Cluster051 is remarkable for containing only genes coding for secreted proteins, with only one from $B$. cinerea and 6 from S. sclerotiorum, such as the SPEP gene SS1G_13371 (Table 3). To get support for the duplication of SS1G_13371 gene ancestor in S. sclerotiorum lineage, we constructed a phylogenetic tree of SS1G_13371 homologs. A total of 58 homologs in 25 fungal species could be retrieved from the JGI database covering 238 complete fungal genomes. We selected the 20 closest homologs to build a parsimony tree based on a 90 amino-acid alignment (Figure 4c). For the $6 \mathrm{~S}$. sclerotiorum genes of cluster 051, the phylogeny revealed clustering based on paralogy rather than orthology, suggesting that this family expanded after the separation of the 25 species analyzed. Gene duplication in this family may have allowed increased accumulation of the corresponding protein, neo-functionalization in some paralogs, or differential regulation.

\section{S. sclerotiorum-specific effector candidates with toxin structural analogs}

The emergence of virulence is frequently associated with a high rate of mutation, gene gain and gene loss in effector genes. This evolutionary pattern results in a discontinuous taxonomic distribution for effector genes $[4,55]$. To identify genes showing a discontinuous taxonomic distribution among $S$. sclerotiorum SPEP genes, we looked for homologs of all 486 S. sclerotiorum SPEP genes in the complete genome of 13 fungal pathogens with a necrotrophic phase of infection covering all major 
Table 2 List of five S. sclerotiorum effector candidates selected based on $\mathrm{Ka} / \mathrm{Ks}>1$ in pairwise comparisons with their B. cinerea orthologs

\begin{tabular}{|c|c|c|c|c|c|c|c|}
\hline Protein ID & Annotation & PFAM domains & Length & $\begin{array}{l}\mathrm{Ka} / \mathrm{Ks} \\
\text { vs BC1T }\end{array}$ & $\begin{array}{l}\text { KaKs vs } \\
\text { BcT4 }\end{array}$ & $\mathrm{Ka} / \mathrm{Ks}>1$ sites & Prob \\
\hline SS1G_04551 & Pectinesterase A & Pectinesterase (PF01095.14) & 308 & 2 & 2 & $\max \mathrm{Ka} / \mathrm{Ks}=0.93$ & NA \\
\hline SS1G_07158 & - & DUF1374 (PF07118) & 328 & 6 & ND & $\max \mathrm{Ka} / \mathrm{Ks}=0.4$ & NA \\
\hline SS1G_07749 & Xylanase & $\begin{array}{l}\text { Glycoside hydrolase } \\
\text { family } 11 \text { (PF00457.12) }\end{array}$ & 200 & 4 & 4 & R36, L43, D74, Q76, S128, N174, S200 & 0.08224 \\
\hline SS1G_10165 & Pectinesterase & Pectinesterase (PF01095.14) & 310 & 2 & 0,061669 & $\begin{array}{l}\text { P22, K31, T34, A36, S54, A63, S85, S86, } \\
\text { G88, S89, Q95, A118, D146, I165, F191, } \\
\text { D208, P211, S212, T213, L218, 226l, 230A, } \\
\text { 233S, 236A, 237G, } 238 \text { T, S246, V253, } \\
\text { M258, S259, N260, } 261 \text { V, N263, V269, } \\
\text { S274, P275, N276, 278Q, H285, A286, } \\
\text { A290, H301, S302, P306, S310, N316, } \\
\text { K318, S319, S324 }\end{array}$ & 0.00038 \\
\hline SS1G_10617 & $\begin{array}{l}\text { Glycoside } \\
\text { hydrolase family } \\
15 \text { protein }\end{array}$ & $\begin{array}{l}\text { Glycoside hydrolase family } \\
15 \text { (PF00723.16); Carbohydrate- } \\
\text { binding module family } 20 \\
\text { (PF00686.14); Carbohydrate-binding } \\
\text { module family } 25 \text { (PF03423.8) }\end{array}$ & 628 & 2 & 0,078172 & $\begin{array}{l}\text { N53, R56, M81, S91, N128, S313, S361, } \\
\text { Q383, Q409, S416, N514, Y526, F537, } \\
\text { V549, K586, V589, S605, S617, Q639 }\end{array}$ & 0.2987 \\
\hline
\end{tabular}

Site specific Ka/Ks ratio estimated using Bayesian inference based on the M8 model of Yang et al., 2000. Only sites with Ka/Ks $>1$ in the mature protein sequence are reported. P-value for positive selection estimated using a likelihood ratio test based on the comparison of twice the log likelihood difference obtained with the M8 and M8a null model with a chi-square probability table of degree of freedom 1. BC1T, Botrytis cinerea B.05 genome; BcT4, Botrytis cinerea T4 genome; ND, not determined.

fungal lineages. As expected, the number of SPEP homologs identified in a given genome decreased with phylogenetic distance to S. sclerotiorum, with 395 SPEPs (81.3\%) having homologs in B. cinerea and only 90 (18.5\%) having homologs in Cryptococcus neoformans (Figure 5a). Forty-six SPEP genes had homologs in all 14 fungal species monitored, and 70 were S. sclerotiorumspecific, absent in the 13 other fungal species considered (Figure 5b). Among those, only six (8.6\%) had weak matches to PFAM domains $(0.004<\mathrm{e}$-value $<0.74)$ other than domains of unknown function. We could not obtain information about the function of the 64 remaining SPEP genes based on annotation or homology.

To get insights into the putative function of S. sclerotiorum-specific predicted SPEPs with no annotation, we performed 3D protein structure predictions followed by fold-recognition searches with an aim to identify structural analogs of known function. We focused on 17 SPEP genes encoding mature proteins of at least 50 amino-acids and for which the best BlastP hit is not a $B$. cinerea protein (Table 4). We closely examined the predicted structures of three SPEPs in comparison with their structural analogs. SS1G_09512 had analogy to the lectin domain of lectinolysin, a toxin forming pores in cell membranes regulated by sugar-binding through a lectin domain [56] (Figure 6a). SS1G_12769 had analogy to the hookworm saposin-like protein Na-SLP-1, a toxin forming pores in membranes through lipid-binding activity [57] (Figure 6b). SS1G_13235 has analogy to the C-terminal domain of death-associated protein 5 (DAP5), a member of the eukaryotic translation initiation factor eIF4G protein family (Figure 6c). Cleavage of DAP5 by caspases at its C-terminus induces apoptosis [58]. SS1G_07354 also had eIF4G protein as closest analog (Table 4). We propose that these EC are toxin analogs that may have emerged through convergent evolution and could contribute to S. sclerotiorum virulence.

\section{S. sclerotiorum effector candidate genes show diverse patterns of expression in planta}

To test whether ECs identified in this work could play a role during interaction with host plants, we monitored the expression pattern after plant inoculation by quantitative RT-PCR for 16 EC genes, representative of the four lists of ECs identified through our bioinformatics analyses. For this, we inoculated four different host and model plants, including Tomato, Nicotiana benthamiana, Arabidopsis thaliana resistant accession Rubezhnoe and susceptible accession Shahdara, and harvested samples at 6, 24 and 48 hours post-inoculation (hpi). S. sclerotiorum mycelium grown in vitro was used as a reference. We included the $S$. sclerotiorum ubiquitin-conjugating enzyme gene SS1G_11173 as non-induced control gene and the cerato-platanin gene SS1G_10096 as an in planta-induced control [14]. Eight effector candidate genes showed in planta induction $>2$ on all four host plants tested. One gene (SS1G_07295) did not show in planta induction $\geq 2$ fold on any plant. Using hierarchical clustering, we grouped effector candidate genes into five clusters according to their expression pattern (Figure 7a). Genes in cluster I show strong induction in planta over at least two time points; genes in cluster II showed strong and early 


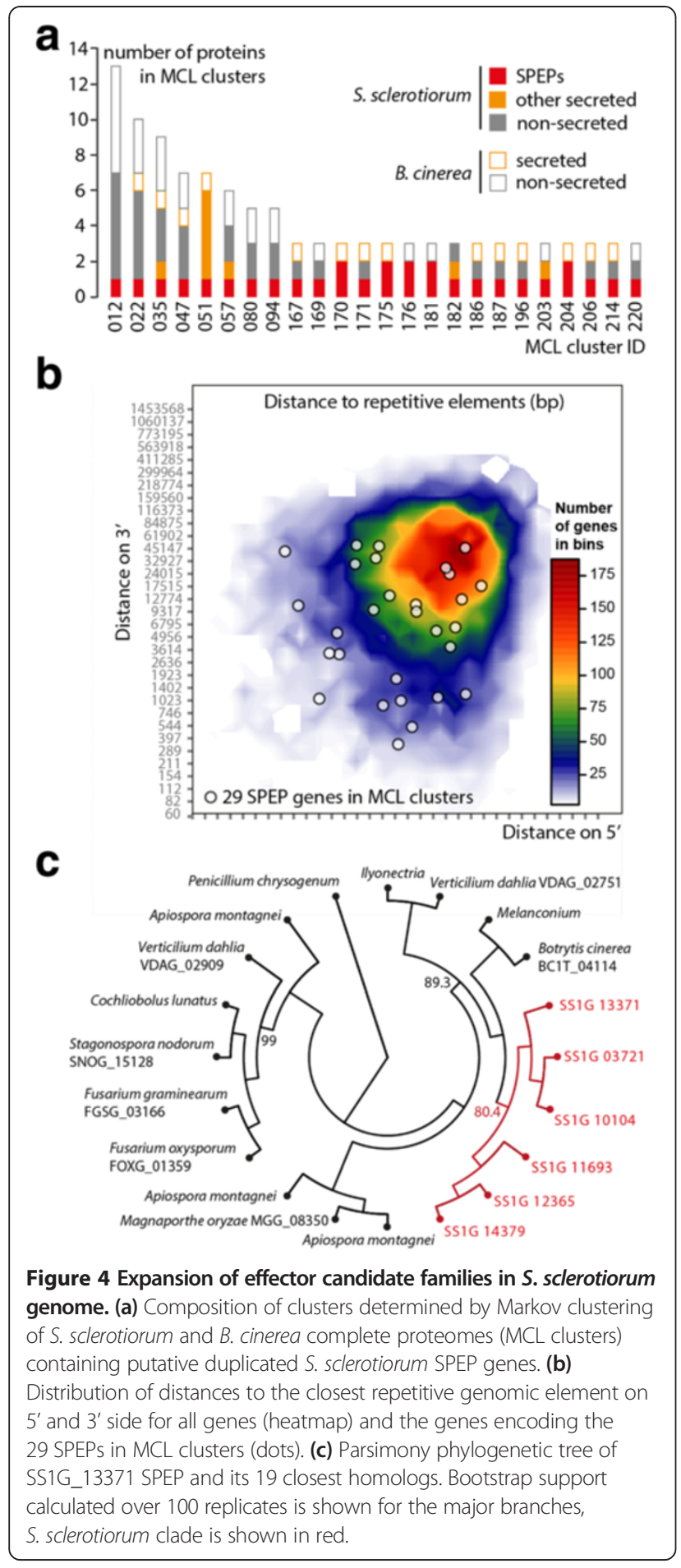

induction in $N$. benthamiana, but only moderate and late induction on other host plants; genes in cluster III showed stronger induction on $A$. thaliana Shahdara accession; genes in cluster IV showed late (48 hpi) induction, stronger on $N$. benthamiana and tomato; and genes in cluster $\mathrm{V}$ showed moderate induction at early time points ( $\leq 24 \mathrm{hpi})$. The cerato-platanin gene SS1G_10096 grouped in cluster IV.

The set of 16 ECs analyzed presented diverse expression patterns. We observed peaks of expression on $N$. benthamiana at 6, 24 or 48 hpi for SS1G_08858, SS1G_07749 and SS1G_00849 respectively illustrating the diversity of induction kinetics. On A. thaliana Shahdara accession, peaks of expression occurred at 6, 24 or 48 hpi for SS1G_01593,SS1G_06213 and SS1G_10096 respectively (Figure $7 \mathrm{~b}$ ). At $24 \mathrm{hpi}$, extensive cell death was visible on leaves of $A$. thaliana accession Shahdara whereas only limited cell death symptoms were visible on $N$. benthamiana (Additional file 2: Figure S1), suggesting that the activation of host cell death is not the only determinant of S. sclerotiorum EC induction. We observed a consistent 2- to 4- fold induction between 6 and $24 \mathrm{hpi}$ for SS1G_06213 on all four host plants tested. By contrast, SS1G_08858 was induced $>4$-fold at $6 \mathrm{hpi}$ on $N$. benthamiana, at $24 \mathrm{hpi}$ on tomato, and at $48 \mathrm{hpi}$ on $A$. thaliana (Figure $7 \mathrm{c}$ ). This result suggests that S. sclerotiorum possess effector genes that are regulated independently of the host being colonized and others that are differentially regulated in a host-dependent manner. Furthermore, SS1G_13371 was induced >2 fold during infection of $A$. thaliana resistant accession Rubezhnoe, but not during infection of the susceptible accession Shahdara. Conversely, SS1G_00849 was induced $>8$ fold during infection of the susceptible accession Shahdara, but only $\sim 4$ fold during infection of the resistant accession Rubezhnoe (Figure 7d). This data points towards a versatile repertoire of effector candidates the expression of which can be modulated according to the nature of the host plant being colonized.

\section{Discussion}

In their global analysis of $S$. sclerotiorum genome, Amselem et al. [12] identified 603 genes encoding non-CAZYme, non-peptidase secreted proteins. These secretome genes did not appear significantly enriched in genes induced in planta. In this study, we combined multiple bioinformatics approaches to identify a total of 745 predicted secreted proteins, among which 486 with experimental evidence for expression in planta (SPEPs). The predicted SPEPs include SsNEP1 and SsITL1 that have proposed to be $S$. sclerotiorum virulence factors $[8,44]$. Since we have chosen to focus the search for effector candidates on these 486 SPEP genes, we have deliberately ignored genes expressed in planta for which experimental evidence is lacking, and enzymes that contribute to the biosynthesis of secondary metabolites as virulence determinants. It is therefore expected that the diversity of $S$. sclerotiorum virulence factors exceeds that of the candidate effectors presented here. Sequence similarity to known fungal effectors is a powerful method to 
Table $\mathbf{3}$ The $\mathbf{2 4}$ gene clusters containing duplicated S. sclerotiorum SPEP genes

\begin{tabular}{|c|c|c|c|c|c|}
\hline Group & $\begin{array}{l}\mathrm{N}^{\circ} \\
\text { Ss }-B c^{*}\end{array}$ & $\begin{array}{l}\text { S. sclerotiorum } \\
\text { SPEPs }\end{array}$ & Other genes in group & SPEPs annotation & $\begin{array}{l}\text { Nearest repeat to } \\
\text { SPEPs (distance } \mathrm{Kb} \text { ) }\end{array}$ \\
\hline Cluster012 & $7-6$ & SS1G_01081 & $\begin{array}{l}\text { BC1G_01968, SS1G_09509, BC1G_01095, SS1G_00547, } \\
\text { BC1G_09386, SS1G_09141, SS1G_05689, BC1G_02407, } \\
\text { SS1G_06186, BC1G_12856, SS1G_02784, BC1G_13021 }\end{array}$ & Catalase & 5SrRNA_AN (41.6) \\
\hline Cluster022 & $6-4$ & SS1G_04468 & $\begin{array}{l}\text { SS1G_04513, SS1G_09104, SS1G_09671, BC1G_12617, } \\
\text { BC1G_09286, SS1G_09338, BC1G_00394, SS1G_14236, } \\
\text { BC1G_00455 }\end{array}$ & $\begin{array}{l}\text { Glycoside hydrolase } \\
\text { family } 47 \text { protein }\end{array}$ & Helitron-2_PSt (47.3) \\
\hline Cluster035 & $5-4$ & SS1G_10949 & $\begin{array}{l}\text { BC1G_01945, SS1G_12508, SS1G_12939, BC1G_02687, } \\
\text { BC1G_11888, BC1G_10788, SS1G_01984, SS1G_14293 }\end{array}$ & Glucose oxidase & BOTY_LTR (0.8) \\
\hline Cluster047 & $4-3$ & SS1G_11700 & $\begin{array}{l}\text { SS1G_08020, BC1G_05350, BC1G_01594, SS1G_11304, } \\
\text { BC1G_11407, SS1G_05897 }\end{array}$ & $\begin{array}{l}\text { Glycoside hydrolase } \\
\text { family } 18 \text { protein }\end{array}$ & BOTY_LTR (1.0) \\
\hline Cluster051 & $6-1$ & SS1G_13371 & $\begin{array}{l}\text { BC1G_04114, SS1G_12365, SS1G_03721, SS1G_14379, } \\
\text { SS1G_11693, SS1G_10104 }\end{array}$ & - & Tad1-14_BG (1.1) \\
\hline Cluster057 & $4-2$ & SS1G_05454 & $\begin{array}{l}\text { BC1G_01964, SS1G_12510, SS1G_00677, SS1G_00773, } \\
\text { BC1G_00533 }\end{array}$ & $\begin{array}{l}\text { Glycosyl hydrolases } \\
\text { family } 18 \text { protein }\end{array}$ & BOTY_LTR (0.9) \\
\hline Cluster080 & $3-2$ & SS1G_05073 & BC1G_10397, SS1G_10773, BC1G_07160, SS1G_13589 & - & BOTY_LTR (5.8) \\
\hline Cluster094 & $3-2$ & SS1G_09630 & SS1G_03681, SS1G_10564, BC1G_10623, BC1G_03527 & $\begin{array}{l}\text { Pyrroline-5-carboxylate } \\
\text { reductase }\end{array}$ & BOTY_LTR (3.6) \\
\hline Cluster167 & $2-1$ & SS1G_13935 & BC1G_12793, SS1G_13934 & CFEM domain containing & BOTY_LTR (9.6) \\
\hline Cluster169 & $2-1$ & SS1G_02369 & SS1G_00501, BC1G_00594 & $\begin{array}{l}\text { Xyloglucan-specific } \\
\text { endo-betaglucanase A }\end{array}$ & BOTY_LTR (0.5) \\
\hline Cluster 170 & $2-1$ & $\begin{array}{l}\text { SS1G_04264, } \\
\text { SS1G_12024 }\end{array}$ & BC1G_15278 & Cell wall glucanase & $\begin{array}{l}\text { BOTY_LTR (13.0) - } \\
\text { Gypsy-31_ADe-I ( } 35.9)\end{array}$ \\
\hline Cluster171 & $2-1$ & SS1G_13501 & BC1G_06328, SS1G_03093 & $\begin{array}{l}\text { Bacterial alpha-L-rhamnosidase } \\
\text { domain protein }\end{array}$ & Harbinger-5_PSt (1.8) \\
\hline Cluster175 & $2-1$ & $\begin{array}{l}\text { SS1G_10092, } \\
\text { SS1G_03618 }\end{array}$ & BC1G_03590 & Endo-beta-xylanase & $\begin{array}{l}\text { BOTY_LTR (3.9) - } \\
\text { BOTY_LTR (11.4) }\end{array}$ \\
\hline Cluster176 & $2-1$ & $\begin{array}{l}\text { SS1G_04958, } \\
\text { SS1G_09225 }\end{array}$ & BC1G_01026 & Tripeptidyl peptidase sed3 & $\begin{array}{l}\text { BOTY_LTR (1.1) - } \\
\text { Mariner-3_AF (5.9) }\end{array}$ \\
\hline Cluster181 & $2-1$ & $\begin{array}{l}\text { SS1G_07498, } \\
\text { SS1G_01811 }\end{array}$ & BC1G_06353 & $\begin{array}{l}\text { Glucose-methanol-choline } \\
\text { oxidoreductase }\end{array}$ & $\begin{array}{l}\text { BOTY_LTR (18.2) - } \\
\text { BOTY_LTR (0.6) }\end{array}$ \\
\hline Cluster182 & $3-1$ & SS1G_12509 & SS1G_00772, SS1G_05453 & LysM domain protein & Mariner-3_AF (8.1) \\
\hline Cluster186 & $2-1$ & SS1G_04200 & BC1G_00245, SS1G_01334 & $\begin{array}{l}\text { Alpha-mannosidase } \\
\text { family protein }\end{array}$ & BOTY_LTR (2.2) \\
\hline Cluster187 & $2-1$ & SS1G_04207 & BC1G_00240, SS1G_04205 & Polygalacturonase & BOTY_LTR (3.4) \\
\hline Cluster196 & $2-1$ & SS1G_05273 & SS1G_11068, BC1G_09997 & Amidase family protein & BOTY_LTR (28.8) \\
\hline Cluster203 & $2-1$ & SS1G_03160 & SS1G_00233, BC1G_07555 & Autophagy related lipase & BOTY_LTR (1.2) \\
\hline Cluster204 & $2-1$ & $\begin{array}{l}\text { SS1G_08110, } \\
\text { SS1G_12361 }\end{array}$ & BC1G_12374 & - & $\begin{array}{l}\text { 5SrRNA_AN (2.3) - } \\
\text { Tad1-14_BG (1.8) }\end{array}$ \\
\hline Cluster206 & $2-1$ & SS1G_08104 & SS1G_09795, BC1G_12379 & Acetyl xylan esterase & 5SrRNA_AN (6.2) \\
\hline Cluster214 & $2-1$ & SS1G_00446 & BC1G_00660, SS1G_05782 & $\begin{array}{l}\text { Heterokaryon incompatibility } \\
\text { Het-c domain protein }\end{array}$ & SINE3-2_AO (5.4) \\
\hline Cluster220 & $2-1$ & SS1G_05461 & BC1G_06146, SS1G_13907 & $\begin{array}{l}\text { Thioesterase-like domain } \\
\text { protein }\end{array}$ & TGATGAA)n (0.3) \\
\hline
\end{tabular}

Repbase nomenclature for transposons used here: BOTY LTR, Botrytis cinerea gypsy-type retrotransposon; Gypsy-31_ADe-I, internal portion of retrotransposon GYPSY31; Harbinger-5_PSt, Harbinger-type DNA transposon; Mariner-3_AF, Mariner DNA transposons; SINE3-2_AO, SINE3 nonautonomous non-LTR retrotransposon; Tad1-14_BG, Tad1 Non-LTR retrotransposon from barley powdery mildew. Retrotransposons are indicated in bold. * Number of genes from S. sclerotiorum - B. cinerea.

uncover effector families conserved across species [16], that allowed us to identify S. sclerotiorum homologs of $B$. cinerea NEP1 [44] and Spl1 [14], M. oryzae MGG_15370 and $C$. hingginsianum CHEC91 and CHEC80. To complement this approach, we used PFAM domain and NLS motif searches to reveal additional effector candidates. We identified putative chitin-binding proteins, putative protease inhibitors, cystein-rich proteins and putative nuclear localized proteins. Effectors with chitin binding activity such as C. fulvum Ecp6, M. oryzae Slp1 and 


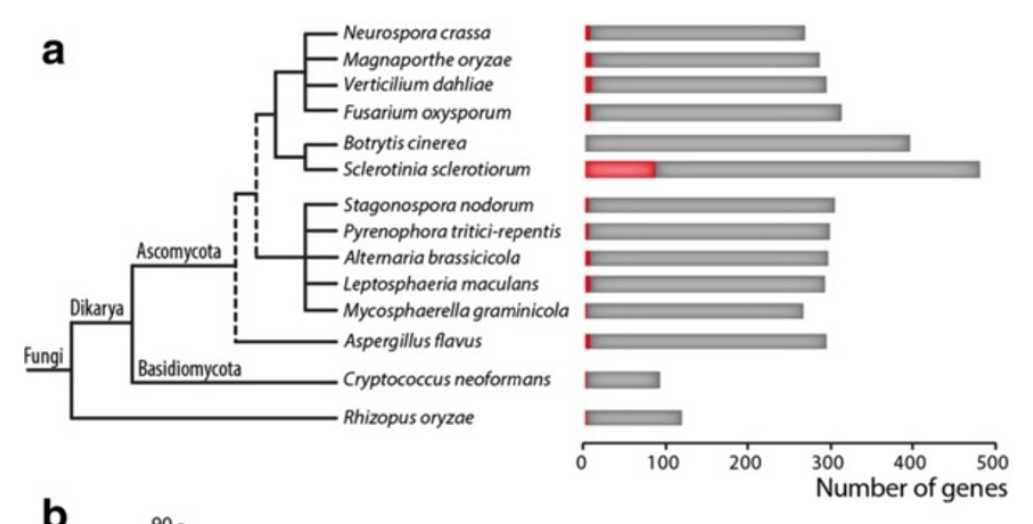

b

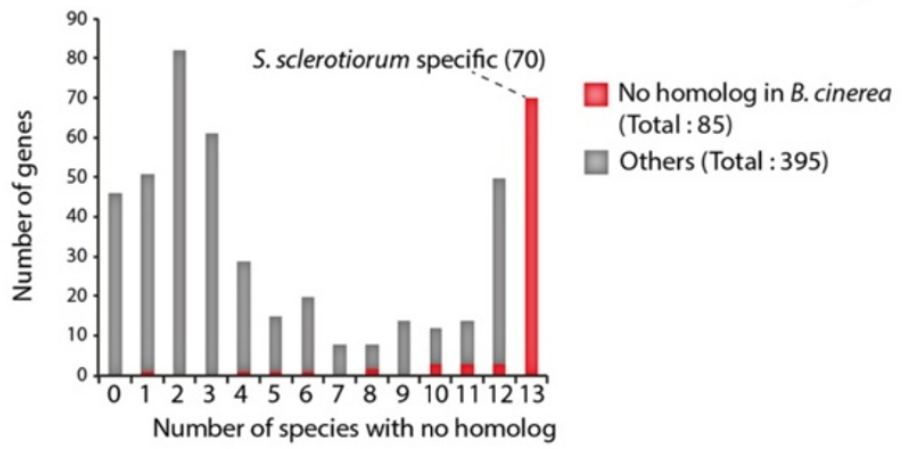

Figure 5 Taxonomic distribution of S. sclerotiorum SPEP genes across 14 fully sequenced fungal pathogen genomes. (a) Bar chart showing the number of S. sclerotiorum SPEP genes conserved along a phylogeny of fungal pathogens. Conservation was determined based on BlastP searches as described in the methods. (b) Distribution of S. sclerotiorum SPEP genes according to the number of species in which they are not conserved. S. sclerotiorum SPEP genes conserved in a given species but not in B. cinerea are shown in red.

M. graminicola Mg3LysM function in suppressing plant immunity [18,20,59]. Similarly, SsITL1 (SS1G_14133) integrin-like secreted protein suppresses plant jasmonic acid and ethylene signaling pathways and enhances susceptibility [8]. These findings suggest that $S$. sclerotiorum secretes proteins able to suppress plant immunity.

The comparative analysis of Fusarium graminearum secretome and genomes of other ascomycetes revealed a high level of conservation with only $25 \mathrm{~F}$. graminearum specific out of 574 secreted proteins [60]. The taxonomic distribution of $S$. sclerotiorum SPEP homologues analyzed in this work supports the conservation of more than 50\% of SPEP genes across ascomycetes. As proposed by Brown et al. [60], these core SPEP genes may support S. sclerotiorum epiphytic growth and highlight important distinctions between multiple phases in infection by this fungus [61]. Nevertheless it also revealed 70 SPEP genes (14\%) specific to $S$. sclerotiorum, many of which are unannotated proteins. The systematic prediction of their 3D structure allowed identifying putative structural analogs of some predicted SPEPs and suggests that they may carry unique functions to assist $S$. sclerotiorum pathogenicity. It will be interesting to take advantage of these predictions to test the biological function of these effector candidates and confront them to experimentally determined structures. Furthermore, in spite of the limited sequence diversity included in the dataset analyzed here, we were able to detect signatures of positive selection in five S. sclerotiorum SPEP genes (2.5\% of genes analyzed). Similar frequency (3.2\%, 21 out of 642 genes) has been reported in B. cinerea [35]. In the future, an in depth exploration of sequence diversity in S. sclerotiorum should allow to reveal more sites subjected to selection. SPEP genes for which positive selection has been detected encode cell wall degrading enzymes, including SS1G_07749 encoding a putative xylanase. This protein is related to $B$. cinerea Xyn11 considered as a Pathogen Associated Molecular Pattern (PAMP) [9]. The detection of positive selection in SS1G_07749 is therefore consistent with the hypothesis that PAMPs may be characterized by signatures of positive selection in a background of strong negative selection [50]. It may therefore be hypothesized that a subset of S. sclerotiorum critical secreted enzymes are engaged in an evolutionary arms race with plant pattern recognition receptors, driving opposing forces of natural selection S. sclerotiorum effector genes. Since plant inhibitors are known for many fungal cell wall degrading enzymes, it is also possible that an evolutionary arms race with plant inhibitors drives the evolution of some S. sclerotiorum effector candidates [53,62,63]. Remarkably, we also identified $14.4 \%$ of species-specific SPEP genes. The extent to which evolutionary constraints imposed by a broad host range contributes to diversification 
Table 4 List of 17 Sclerotinia-specific effector candidates revealed by pattern and fold recognition searches

\begin{tabular}{|c|c|c|c|c|c|}
\hline & Length & C-score & TM score & Analog model & Selected analog description \\
\hline SS1G_00780 & 115 & -3.14 & 0.610 & 2PFV & S. cerevisiae Exo70 with additional residues to 2.1 Angstrom resolution \\
\hline SS1G_01817 & 116 & -2.53 & 0.503 & $2 \mathrm{R} 5 \mathrm{M}$ & $\begin{array}{l}\text { Crystal Structure of the two MBT repeats from Sex-Comb on Midleg } \\
\text { (SCM) in complex with peptide R-(me)K-S }\end{array}$ \\
\hline SS1G_03830 & 155 & -4.41 & 0.460 & $3 \mathrm{GH} 1$ & Predicted nucleotide-binding protein from Vibrio cholerae \\
\hline SS1G_03878 & 132 & -3.79 & 0.645 & $1 \mathrm{NI3}$ & Schizosaccharomyces pombe YchF GTPase \\
\hline SS1G_04309 & 177 & -4.63 & 0.461 & 2QPD & $\begin{array}{l}\text { Crystal structure of SusD-like carbohydrate binding protein } \\
\text { (YP_001298396.1) from }\end{array}$ \\
\hline SS1G_06504 & 93 & -2.38 & 0.503 & $4 \mathrm{HOI}$ & Bacteroides vulgatus ATCC 8482 at 1.70 A resolution \\
\hline SS1G_07354 & 129 & -3.63 & 0.589 & $1 \cup G 3$ & $\begin{array}{l}\text { Crystal structure of PAS domain from the mouse EAG1 potassium } \\
\text { channel C-terminal portion of human elF4GI }\end{array}$ \\
\hline SS1G_07543 & 273 & -4.27 & 0.706 & $2 \mathrm{FX} 5$ & Pseudomonas mendocina lipase \\
\hline SS1G_08860 & 141 & -3.96 & 0.526 & $1 X L Y$ & X-ray structure of the RNA-binding protein SHE2p \\
\hline SS1G_09512 & 164 & -3.65 & 0.546 & 3LEO & Lectin Domain of Lectinolysin complexed with Glycerol \\
\hline SS1G_10915 & 136 & -4.12 & 0.512 & $3 \mathrm{M} 1 \mathrm{C}$ & $\begin{array}{l}\text { Crystal structure of the conserved herpesvirus fusion regulator } \\
\text { complex } \mathrm{gH}-\mathrm{gL}\end{array}$ \\
\hline SS1G_11461 & 114 & -3.2 & 0.526 & $2 \mathrm{HP3}$ & $\begin{array}{l}\text { Penicillin-binding protein } 2 b \text { (PBP-2b) from Streptococcus } \\
\text { pneumoniae (strain 5204) }\end{array}$ \\
\hline SS1G_12769 & 88 & -2.32 & 0.585 & 3563 & Saposin-like protein Na-SLP-1 \\
\hline SS1G_13016 & 124 & -2.76 & 0.555 & 3APO & $\begin{array}{l}\text { ERAD pathway mediated by the ER-resident protein disulfide } \\
\text { reductase ERdj5 }\end{array}$ \\
\hline SS1G_13142 & 131 & -4.25 & 0.506 & 1DCU & Oxidized pea fructose-1,6-bisphosphate phosphatase \\
\hline SS1G_13235 & 114 & -3.55 & 0.585 & $3 \mathrm{D} 3 \mathrm{M}$ & C-terminal region of Death Associated Protein 5(DAP5) \\
\hline SS1G_14000 & 159 & -4.43 & 0.578 & $2 \mathrm{C} 9 \mathrm{~K}$ & $\begin{array}{l}\text { Structure of the functional form of the mosquito larvicidal } \\
\text { Cry4Aa toxin from Bacillus thuringiensis at a 2.8-angstrom resolution }\end{array}$ \\
\hline
\end{tabular}

C-score is a confidence score for estimating the quality of models predicted by I-TASSER ranging from -5 (low confidence) to 2 (high confidence). TM score is a quality score for the superimposition of 3D models calculated with TM-align with values in [0;1]. A TM-score $>0.5$ generally corresponds to the same fold in SCOP/CATH.

in the effector candidate repertoire of $S$. sclerotiorum remains to be determined. Detailed functional analysis of effector gene alleles and their plant targets will be needed to address this question.

Although S. sclerotiorum is considered as a typical necrotroph, there is evidence that it colonizes plant tissues through multiple phases involving important transcriptional and physiological reprogramming [61]. Consistent with this model, the phytotoxin oxalic acid dampens plant immune responses at the initial stages of infection and later enhances programmed cell death [7]. In this work, we report the sequential transcriptional activation of S. sclerotiorum candidate effector genes. A $>2$ fold induction was measured at $6 \mathrm{hpi}$ for several effector candidate genes, whereas no necrotic symptoms are visible at this time, except on A. thaliana Shahdara accession. This suggests that the sequential secretion of effectors is required for the efficient induction of host cell death by $S$. sclerotiorum, or that some secreted proteins could contribute to $S$. sclerotiorum virulence independently of host cell death activation. By comparing the expression pattern of selected Blumeria graminis $\mathrm{f}$. sp. hordei genes grown on barley and on A. thaliana,
Hacquard et al. [64] concluded that very divergent hosts do not significantly alter the fungal gene expression program. The expression pattern of some S. sclerotiorum ECs is indeed independent on the host plant being colonized (e.g. SS1G_06213). Nevertheless, other ECs showed differential regulation in a host-dependent manner (e.g. SS1G_08858, SS1G_13371). The transcriptional activation of distinct set of effectors depending on the host being colonized has also been reported for the generalist root endophyte Piriformospora indica in barley and A. thaliana [65]. The growing number of transcriptomic studies on various pathosystems should help determine whether host-dependent modulation of effector gene expression differs according to pathogens lifestyle or host range. We speculate that the white mold fungus benefits from a versatile repertoire of secreted proteins with diverse functions, evolution and expression patterns, to successfully infect a wide range of host plants. A systematic characterization of S. sclerotiorum transcriptome on multiple hosts and the functional analysis of differentially regulated effector genes should prove useful to decipher the molecular determinants of quantitative disease resistance and host range in this fungal pathogen. 


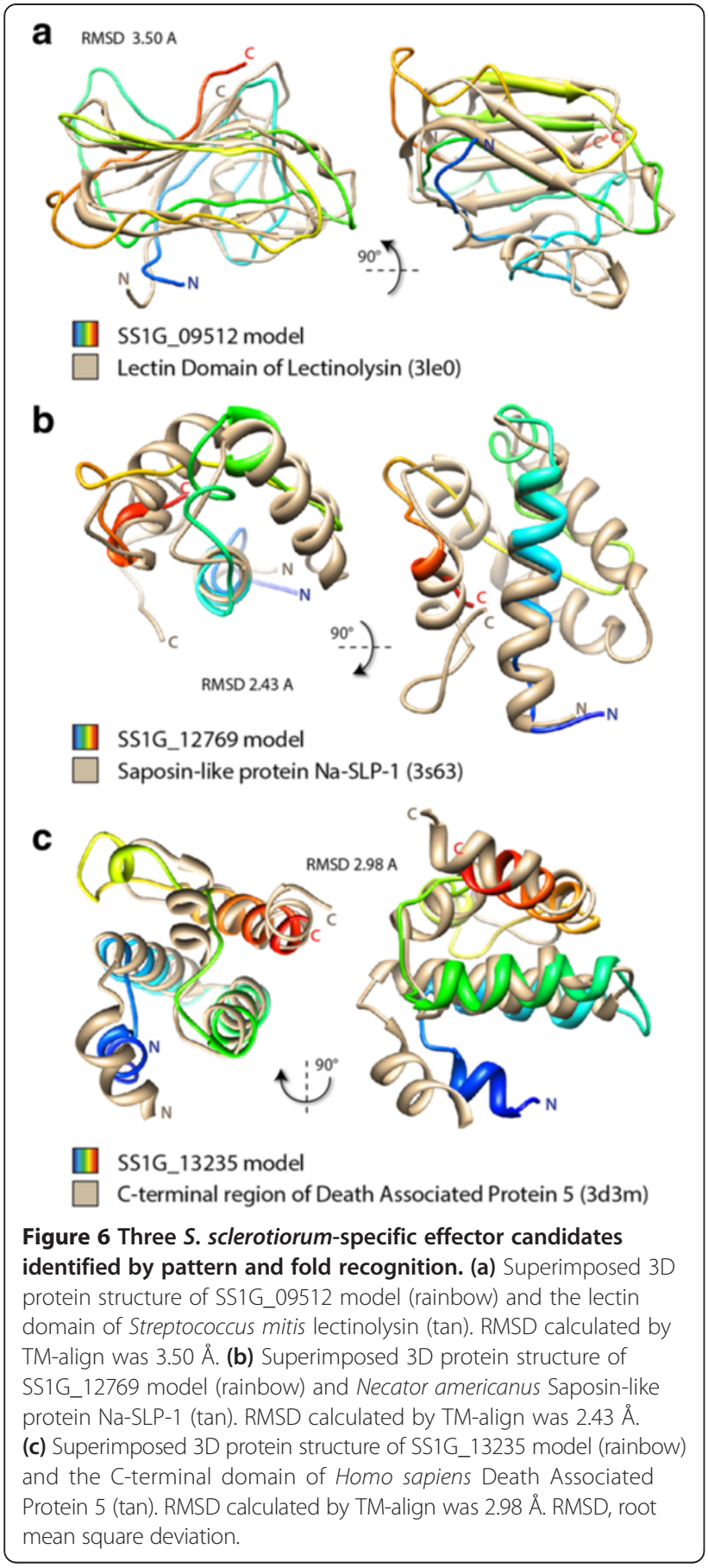

\section{Conclusions}

In this work, we explored systematically the diversity of candidate virulence genes in the necrotrophic fungal pathogen S. sclerotiorum using in silico structure and evolution analyses. We report the identification of $486 \mathrm{~S}$. sclerotiorum secreted proteins expressed in planta, including 78 ECs. We have analyzed in planta expression for a representative subset of 16 ECs, highlighting diverse predicted functions and expression patterns. This study reveals that besides plant degrading enzymes, $S$. sclerotiorum genome encodes numerous predicted secreted proteins that may be involved in the interaction between the fungus and its host plants. It will facilitate future investigation on their relevance in the infection process and sheds new light on the underestimated complexity of host colonization by necrotrophic plant pathogens.

\section{Methods}

\section{Secretome prediction and annotation}

We used complete genome and predicted proteomes of Sclerotinia sclerotiorum strain 1980 v.2, Botrytis cinerea strain b05.10 v.1 and strain t4 v.1 described in [12]. The presence of secretion signals was predicted with SignalP v.2 and v.4 [66,67], transmembrane helices and GPI anchor sequence were predicted with TMHMM [68] and GPIsom [69] respectively. For the identification of genes expressed in planta, microarrays data for gene induction fold at 2 days post inoculation on sunflower cotyledons and Expressed Sequence Tags (ESTs) from [12] were used. ESTs were assigned to the $S$. sclerotiorum predicted transcript giving the lowest e-value in a BLASTN search. Genes were considered expressed in planta when either (i) showing induction fold $\geq 1$ in during sunflower infection in microarrays data or (ii) being assigned at least one EST in either infection cushion, infected $B$. napus or infected tomato library. S. sclerotiorum predicted proteins were annotated using Blast2GO [70], PFAM [71] and NLStradamus [72]. Predicted proteins shorter than 40 amino-acids were excluded from the analysis. PFAM domains were annotated using HMMER3 searches against the PFAM 26.0 database [71]. We defined non-annotated predicted SPEPs as having no hit to PFAM_A with e-value $<0.1$. For the identification proteins similar to known fungal effectors, BlastP searches against a local database of 191 effectors with an e-value cutoff of $1 \mathrm{e}^{-3}$.

\section{Definition of ortholog clusters and natural selection analysis} Core ortholog groups (COGs) between S. sclerotiorum 1980 and B. cinerea b05.10 or B. cinerea t4 proteomes were identified using Inparanoid7 [73] with the following parameters: score cutoff 40 bits; sequence overlap cutoff 0.5 ; group merging cutoff 0.5 ; scoring matrix BLOSUM62. COGs in which a length difference $>10$ aminoacids existed between $S$. sclerotiorum and B. cinerea were discarded. Pairwise ortholog alignments were generated using the needleall program from the EMBOSS package using the following parameters: gapopen 50.0; gapextend 0.2 ; minscore 100.0; aformat3 MARKX3. Needleall output files were parsed into .axt alignments used as input in $\mathrm{Ka} / \mathrm{Ks}$ calculator2 [74]. $\mathrm{Ka} / \mathrm{Ks}$ ratios were 


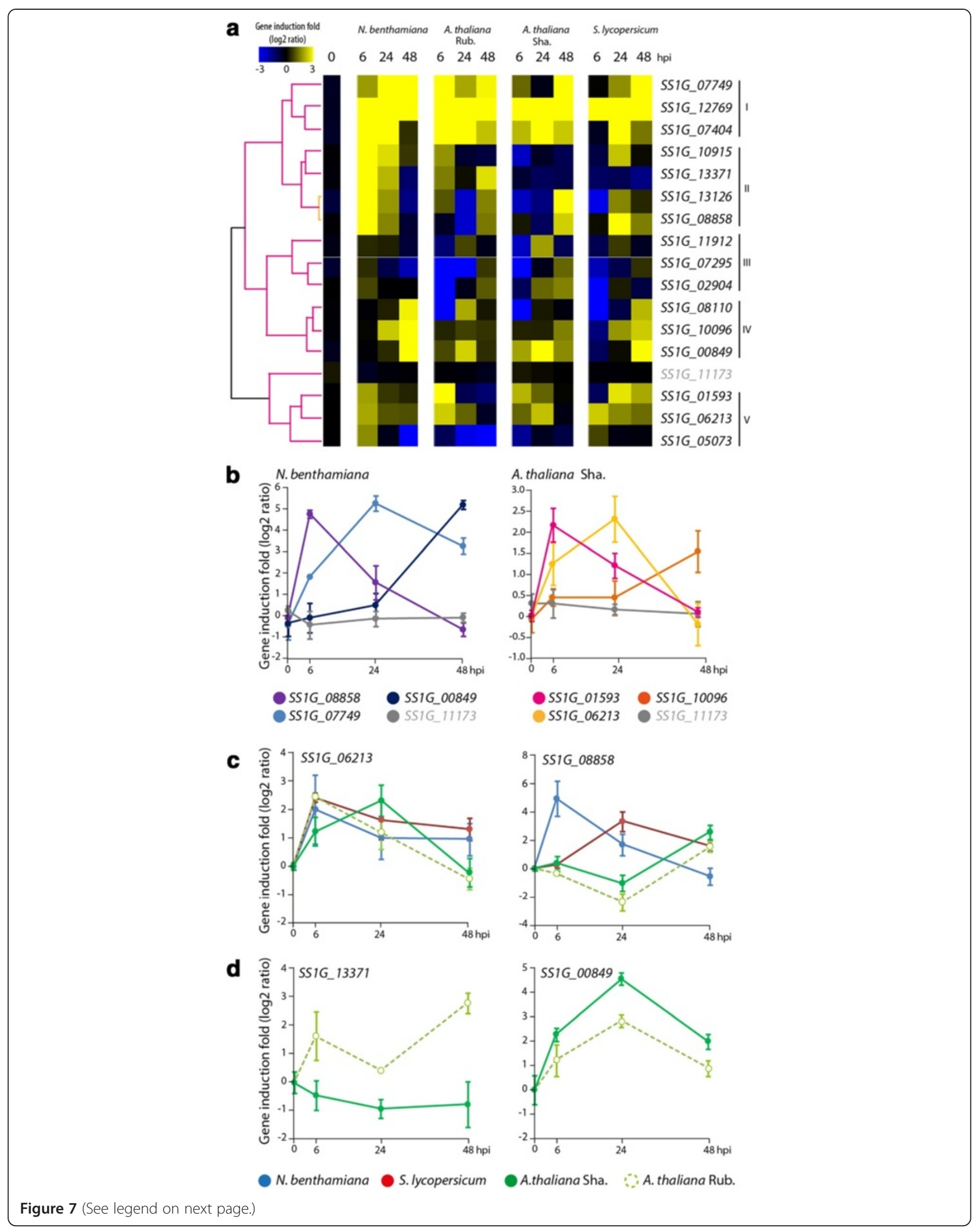


(See figure on previous page.)

Figure 7 In planta expression analysis for selected S. sclerotiorum effector candidates on four different hosts. (a) Transcriptional profiles of 16 S. sclerotiorum effector candidate genes. Overrepresented (yellow) and underrepresented transcripts (blue) in planta are shown as log2-fold changes relative to expression in vitro, normalized using Actin expression. Hierarchical clustering based on Pearson correlation coefficients delimited five clusters. The SS1G_11173 ubiquitin 16 gene was used as a non-induced control. (b) Sequential transcriptional activation of effector gene candidates during the infection of $\mathrm{N}$. benthamiana (left) and A. thaliana Sha. accession (right). (c) In planta expression pattern of candidate effector genes showing host-independent expression (SS1G_06213, left) and host-dependent expression (SS1G_08858, right). (d) Differential expression patterns of two candidate effector genes on susceptible (Sha.) and resistant (Rub.) A. thaliana accessions. Relative gene expression shown as log2-fold changes relative to expression in vitro, normalized using Actin expression. Error bars show standard deviation calculated from two technical replicates on each of three independent biological experiments. Rub., Rubezhnoe; Sha., Shahdara.

calculated for all COG pairs using Yn00 method [28]. The identification of codon sites under positive selection was achieved through Bayesian inference using the Selecton2.2 server [75] with the "Positive selection enabled (M8, beta $+\mathrm{w}>=1$ )" evolutionary model with 8 categories, on alignments of S. sclerotiorum 1980, B. cinerea b05.10 and B. cinerea $\mathrm{t} 4$ orthologs.

\section{Protein structure modeling and analysis}

Protein structure modeling was performed with the I-TASSER server [76] and rendered using UCSF Chimera [77]. Site-specific alignment consensus and $\mathrm{Ka} / \mathrm{Ks}$ ratios were mapped onto protein models using the 'define attribute' function in UCSF Chimera. Moving average over a 3 amino-acid window of the percentage consensus in a 99 homologous protein alignment was used to characterize conservation in SS1G_01593 family. Structural analogs were identified using the TM-align program in I-TASSER.

\section{Taxonomic distribution and phylogenetic analyses}

Fungal taxonomy trees are based on [16]. The presence of SPEP homologs in 234 fungal species was assessed using BlastP searches against JGI fungi Gene Catalog Proteins [78] with an e-value cutoff of $1 \mathrm{e}^{-5}$ without low complexity filter. Among retrieved homologs, proteins that had no signal peptide detected with SignalP4 or SignalP2 were discarded. For the global analysis of taxonomic distribution of SPEP genes, the predicted proteomes of Neurospora crassa, Magnaporthe oryzae, Verticilium dahlia, Fusarium oxysporum, Stagonospora nodorum, Pyrenophora tritici-repentis, Alternaria brassicicola, Leptosphaeria maculans, Mycosphaerella graminicola, Aspergillus flavus, Cryptococcus neoformans and Rhizopus oryzae were used in local BlastP searches with e-value cutoff 1 . For each SPEP gene, BlastP scores for all hits in a given species were summed up, and SPEP genes were considered as absent if total score is $<2$. Phylogenetic trees for SS1G_13371 family was generated using the parsimony method with 100 bootstrap replicates with the Extended Majority rule, as implemented in the protpars and consense programs of the Phylip 3.67 package [79].

\section{Sequence-based clustering and genome distribution of duplicated genes}

S. sclerotiorum 1980 and B. cinerea b05.10 proteins were clustered based on sequence similarity by Markov clustering using the orthoMCL function in Biolayout 3D [80]. A self BlastP search on the combined S. sclerotiorum 1980 and B. cinerea b05.10 complete proteomes with e-value cutoff $1 \mathrm{e}^{-30}$ was used as input for orthoMCL. Repeats and transposable elements were identified using RepeatMasker on S. sclerotiorum 1980 supercontigs with the cross_match method at slow speed and "Fungi" as a DNA source. Genomic distances and genome architecture heatmaps were generated according to [81].

\section{Plant and fungus cultivation, inoculation procedure}

Arabidopsis thaliana accession Shahdara and Rubezhnoe1 were grown in Jiffy pots for four weeks at $22^{\circ} \mathrm{C}$ with cycles of 9 hours of light per 24 hours. Tomato (Solanum lycopersicum cv. Heinz) were grown for six weeks in pots containing disinfected soil in a greenhouse at $23^{\circ} \mathrm{C}$ with cycles of $\sim 14$ hours of light per 24 hours. Nicotiana benthamiana plants were grown for four weeks at $21^{\circ} \mathrm{C}$ with cycles of 16 hours of light per 24 hours. S. sclerotiorum strain S55 was first grown for 4 days on PDA plates at $25^{\circ} \mathrm{C}$ in the dark. Fifty $\mathrm{mL}$ of liquid PDB medium were inoculated with 3 agar plugs of PDA cultures and incubated for 4 days at $25^{\circ} \mathrm{C}$ in the dark, with $150 \mathrm{rpm}$ shaking. Three independent inoculation experiments were performed in which fully grown plant leaves were cut and placed right side up on a wet paper towel in large petri dishes. Mycelium was washed twice in PDB, filtered on Miracloth (Calbiochem, CA), and spread over whole leaf surfaces. Inoculated leaves were incubated for up to 3 days at $25^{\circ} \mathrm{C}$ with 14 hours of light per 24 hours.

\section{Effector candidate gene expression by quantitative RT-PCR}

Plant leaves were harvested immediately and 6, 24 and 48 hours after inoculation, and ground in liquid nitrogen. Total RNA was extracted using a Nucleospin RNA II kit (Machery- Nagel) according to manufacturer's instructions. RNAs were analyzed and quantified on an Agilent 2100 Bioanalyzer. The first-strand cDNA was synthesized using TRT reverse transcriptase (Roche) according to 
manufacturer's instructions. Real-time PCR reactions included $3.5 \mu \mathrm{L}$ of SYBR green mix (Roche), $1 \mu \mathrm{L}$ of $5 \mu \mathrm{M}$ primers (Additional file 3: Table S2) and $200 \mathrm{ng}$ of cDNA. Reactions were performed on a Light Cycler 480 II machine (Roche) under the following conditions: $95^{\circ} \mathrm{C}$ for 5 minutes; 45 cycles of $95^{\circ} \mathrm{C}$ for 15 seconds, $65^{\circ} \mathrm{C}$ for 20 seconds and $72^{\circ} \mathrm{C}$ for 20 seconds; then $95^{\circ} \mathrm{C}$ for $10 \mathrm{sec}-$ onds; $65^{\circ} \mathrm{C}$ for 15 seconds followed by a progressive in increase in temperature at $0.11^{\circ} \mathrm{C} /$ second up to $95^{\circ} \mathrm{C}$ to obtain melt curve. S. sclerotiorum actin (SS1G_08733) and ubiquitin 16 (SS1G_11173) genes were used as controls. The expression of effector gene candidates relative to $\mathrm{Ct}$ values of the control genes was determined and analyzed using the LightCycler 480 SW 1.5 software. Fungal cultures were grown in vitro for 3 days and either harvested immediately (Day 0) or inoculated to plants. Values are given as $\log 2$ ratio over Day 0 expression. Error bars represent standard deviation calculated from two technical replicates on each of three independent biological experiments.

\section{Additional files}

Additional file 1: Table S1. Summary table of annotations for the 486 SPEP genes, including homology, domain and motif searches and expression data. Inclusion into sequence-based clusters, into COG groups, $\mathrm{Ka} / \mathrm{Ks}$ values and closest structural analogs are indicated where relevant.

Additional file 2: Figure S1. Representative symptoms of detached leaves inoculated by S. sclerotiorum S55 at 6,24 and 48 hours post inoculation (hpi)

Additional file 3: Table S2. List of primers used in this work.

\section{Competing interest}

The authors declare that they have no competing interests.

\section{Authors' contributions}

KG and CB performed experiments and analyzed transcriptomic data. KG and SR performed secretome analysis. SR designed the research. DR and SR wrote the manuscript, all authors commented on the manuscript before submission. All authors read and approved the final manuscript.

\section{Acknowledgements}

SR is supported by a Marie Curie CIG grant ("SEPAraTE", contract 334036) and a starting grant of the European Research Council ("VariWhim", contract 336808). This work was supported by the French Laboratory of Excellence project "TULIP" (ANR-10-LABX-41; ANR-11-IDEX-0002-02).

Received: 3 February 2014 Accepted: 27 April 2014

Published: 4 May 2014

\section{References}

1. Peltier AJ, Bradley CA, Chilvers MI, Malvick DK, Mueller DS, Wise KA, Esker PD: Biology, yield loss and control of Sclerotinia stem rot of soybean. J Integrated Pest Manage 2012, 3(2):B1-B7.

2. Bolton MD, Thomma BPHJ, Nelson BD: Sclerotinia sclerotiorum (Lib.) de Bary: biology and molecular traits of a cosmopolitan pathogen. Mol Plant Pathol 2006, 7(1):1-16.

3. Rafiqi M, Ellis JG, Ludowici VA, Hardham AR, Dodds PN: Challenges and progress towards understanding the role of effectors in plant-fungal interactions. Curr Opin Plant Biol 2012, 15(4):477-482.

4. Raffaele S, Kamoun S: Genome evolution in filamentous plant pathogens: why bigger can be better. Nat Rev Microbiol 2012, 10(6):417-430.
5. Lorang JM, Sweat TA, Wolpert TJ: Plant disease susceptibility conferred by a "resistance" gene. Proc Natl Acad Sci 2007, 104(37):14861.

6. Lorang J, Kidarsa T, Bradford C, Gilbert B, Curtis M, Tzeng SC, Maier C, Wolpert T: Tricking the Guard: Exploiting Plant Defense for Disease Susceptibility. Sci Signal 2012, 338(6107):659.

7. Williams B, Kabbage M, Kim HJ, Britt R, Dickman MB: Tipping the balance: Sclerotinia sclerotiorum secreted oxalic acid suppresses host defenses by manipulating the host redox environment. PLoS Pathog 2011, 7(6):e1002107

8. Zhu W, Wei W, Fu Y, Cheng J, Xie J, Li G, Yi X, Kang Z, Dickman MB, Jiang D: A Secretory Protein of Necrotrophic Fungus Sclerotinia sclerotiorum That Suppresses Host Resistance. PLoS One 2013, 8(1):e53901.

9. Noda J, Brito N, González C: The Botrytis cinerea xylanase Xyn11A contributes to virulence with its necrotizing activity, not with its catalytic activity. BMC plant Biol 2010, 10(1):38.

10. Lowe RG, Howlett BJ: Indifferent, affectionate, or deceitful: lifestyles and secretomes of fungi. PLoS Pathog 2012, 8(3):e1002515.

11. Saunders DGO, Win J, Cano LM, Szabo LJ, Kamoun S, Raffaele S: Using Hierarchical Clustering of Secreted Protein Families to Classify and Rank Candidate Effectors of Rust Fungi. PLoS One 2012, 7(1):e29847.

12. Amselem J, Cuomo CA, van Kan JAL, Viaud M, Benito EP, Couloux A, Coutinho PM, de Vries RP, Dyer PS, Fillinger S: Genomic Analysis of the Necrotrophic Fungal Pathogens Sclerotinia sclerotiorum and Botrytis cinerea. PLoS Genet 2011, 7(8):e1002230.

13. Ottmann C, Luberacki B, Kufner I, Koch W, Brunner F, Weyand M, Mattinen L, Pirhonen M, Anderluh G, Seitz HU, HU Nurnberger T, Oecking C: A common toxin fold mediates microbial attack and plant defense. Proc Natl Acad Sci U S A 2009, 106(25):10359-10364.

14. Frías M, González C, Brito N: BcSpl1, a cerato-platanin family protein, contributes to Botrytis cinerea virulence and elicits the hypersensitive response in the host. New Phytol 2011, 192:483-495.

15. Koharudin LM, Viscomi AR, Jee J-G, Ottonello S, Gronenborn AM: The evolutionarily conserved family of cyanovirin-N homologs: structures and carbohydrate specificity. Structure 2008, 16(4):570-584.

16. Stergiopoulos I, Kourmpetis YA, Slot JC, Bakker FT, De Wit PJ, Rokas A: In silico characterization and molecular evolutionary analysis of a novel superfamily of fungal effector proteins. Mol Biol Evol 2012, 29(11):3371-3384

17. Van den Burg HA, Westerink N, Francoijs KJ, Roth R, Woestenenk E, Boeren S, de Wit PJGM, Joosten MHAJ, Vervoort J: Natural disulfide bond-disrupted mutants of AVR4 of the tomato pathogen Cladosporium fulvum are sensitive to proteolysis, circumvent Cf-4-mediated resistance, but retain their chitin binding ability. J Biol Chem 2003, 278(30):27340-27346.

18. de Jonge R, van Esse HP, Kombrink A, Shinya T, Desaki $Y$, Bours R, van der Krol S, Shibuya N, Joosten MH, Thomma BP: Conserved fungal LysM effector Ecp6 prevents chitin-triggered immunity in plants. Science 2010, 329(5994):953-955.

19. Marshall R, Kombrink A, Motteram J, Loza-Reyes E, Lucas J, Hammond-Kosack $K E$, Thomma BP, Rudd JJ: Analysis of two in planta expressed LysM effector homologs from the fungus Mycosphaerella graminicola reveals novel functional properties and varying contributions to virulence on wheat. Plant Physiol 2011, 156(2):756-769.

20. Mentlak TA, Kombrink A, Shinya T, Ryder LS, Otomo I, Saitoh H, Terauchi R, Nishizawa Y, Shibuya N, Thomma BP: Effector-mediated suppression of chitin-triggered immunity by Magnaporthe oryzae is necessary for rice blast disease. Plant Cell Online 2012, 24(1):322-335.

21. Rooney HC, van't Klooster JW, van der Hoorn RA, Joosten MH, Jones JD, de Wit PJ: Cladosporium Avr2 inhibits tomato Rcr3 protease required for Cf-2-dependent disease resistance. Science 2005, 308(5729):1783-1786.

22. Mueller AN, Ziemann S, Treitschke S, Aßmann D, Doehlemann G: Compatibility in the Ustilago maydis-maize interaction requires inhibition of host cysteine proteases by the fungal effector Pit2. PLoS Pathog 2013, 9(2):e1003177.

23. Pretsch K, Kemen A, Kemen E, Geiger M, Mendgen K, Voegele R: The rust transferred proteins - a new family of effector proteins exhibiting protease inhibitor function. Mol Plant Pathol 2013, 14(1):96-107.

24. Catanzariti AM, Dodds PN, Lawrence GJ, Ayliffe MA, Ellis JG: Haustorially expressed secreted proteins from flax rust are highly enriched for avirulence elicitors. Plant Cell 2006, 18(1):243-256. 
25. Orbach MJ, Farrall L, Sweigard JA, Chumley FG, Valent B: A telomeric avirulence gene determines efficacy for the rice blast resistance gene Pi-ta. Plant Cell 2000, 12(11):2019-2032.

26. Djamei A, Schipper K, Rabe F, Ghosh A, Vincon V, Kahnt J, Osorio S, Tohge T, Fernie AR, Feussner I, Feussner K, Meinicke P, Stierhof YD, Schwarz H, Macek B, Mann M, Kahmann R: Metabolic priming by a secreted fungal effector. Nature 2011, 478(7369):395-398.

27. Hemetsberger C, Herrberger C, Zechmann B, Hillmer M, Doehlemann G: The Ustilago maydis effector Pep1 suppresses plant immunity by inhibition of host peroxidase activity. PLoS Pathog 2012, 8(5):e1002684.

28. Yang Z, Nielsen R: Estimating synonymous and nonsynonymous substitution rates under realistic evolutionary models. Mol Biol Evol 2000, 17(1):32-43.

29. Win J, Morgan W, Bos J, Krasileva KV, Cano LM, Chaparro-Garcia A, Ammar R, Staskawicz BJ, Kamoun S: Adaptive evolution has targeted the C-terminal domain of the RXLR effectors of plant pathogenic oomycetes. Plant Cell 2007, 19(8):2349-2369.

30. Hacquard S, Joly DL, Lin Y-C, Tisserant E, Feau N, Delaruelle C, Legué V, Kohler A, Tanguay $P$, Petre B, Frey $P$, Van de Peer $Y$, Rouzé $P$, Martin F, Hamelin RC, Duplessis S: A comprehensive analysis of genes encoding small secreted proteins identifies candidate effectors in Melampsora larici-populina (poplar leaf rust). Mol Plant-Microbe Interact 2012, 25(3):279-293.

31. Sun G, Yang Z, Kosch $T$, Summers $K$, Huang J: Evidence for acquisition of virulence effectors in pathogenic chytrids. BMC Evol Biol 2011, 11(1):195.

32. Stergiopoulos I, De Kock MJD, Lindhout P, De Wit PJGM: Allelic variation in the effector genes of the tomato pathogen Cladosporium fulvum reveals different modes of adaptive evolution. Mol Plant-Microbe Interact 2007, 20 (10):1271-1283.

33. Barrett LG, Thrall PH, Dodds PN, van der Merwe M, Linde CC, Lawrence GJ, Burdon JJ: Diversity and evolution of effector loci in natural populations of the plant pathogen Melampsora lini. Mol Biol Evol 2009, 26(11):2499-2513.

34. Cantu D, Segovia V, MacLean D, Bayles R, Chen X, Kamoun S, Dubcovsky J, Saunders DG, Uauy C: Genome analyses of the wheat yellow (stripe) rust pathogen Puccinia striiformis f. sp. tritici reveal polymorphic and haustorial expressed secreted proteins as candidate effectors. BMC Genomics 2013, 14(1):270.

35. Aguileta G, Lengelle J, Chiapello H, Giraud T, Viaud M, Fournier E, Rodolphe F, Marthey S, Ducasse A, Gendrault A: Genes under positive selection in a model plant pathogenic fungus, Botrytis. Infect Genet Evol 2012, 12(5):987-996.

36. Martinez JP, Oesch NW, Ciuffetti LM: Characterization of the multiple-copy host-selective toxin gene, ToxB, in pathogenic and nonpathogenic isolates of Pyrenophora tritici-repentis. Mol Plant-Microbe Interact 2004, 17(5):467-474

37. Manning VA, Pandelova I, Dhillon B, Wilhelm LJ, Goodwin SB, Berlin AM, Figueroa M, Freitag M, Hane JK, Henrissat B: Comparative genomics of a plant-pathogenic fungus, Pyrenophora tritici-repentis, reveals transduplication and the impact of repeat elements on pathogenicity and population divergence. G3 Genes| Genomes| Genetics 2013, 3(1):41-63.

38. Raffaele S, Farrer RA, Cano LM, Studholme DJ, MacLean D, Thines M, Jiang RHY, Zody MC, Kunjeti SG, Donofrio NM: Genome evolution following host jumps in the Irish potato famine pathogen lineage. Science 2010, 330(6010):1540.

39. Rouxel T, Grandaubert J, Hane JK, Hoede C, van de Wouw AP, Couloux A, Dominguez V, Anthouard V, Bally P, Bourras S: Effector diversification within compartments of the Leptosphaeria maculans genome affected by Repeat-Induced Point mutations. Nat Commun 2011, 2:202.

40. Desveaux D, Singer AU, Dangl JL: Type III effector proteins: doppelgangers of bacterial virulence. Curr Opin Plant Biol 2006, 9(4):376-382.

41. Whisson SC, Boevink PC, Moleleki L, Avrova AO, Morales JG, Gilroy EM, Armstrong MR, Grouffaud S, van West P, Chapman S, Hein I, Toth IK, Pritchard L, Birch PR: A translocation signal for delivery of oomycete effector proteins into host plant cells. Nature 2007, 450(7166):115-118.

42. Godfrey D, Böhlenius H, Pedersen C, Zhang Z, Emmersen J, ThordalChristensen $\mathrm{H}$ : Powdery mildew fungal effector candidates share N-terminal Y/F/WxC-motif. BMC Genomics 2010, 11(1):317.

43. Choo KH, Tan TW, Ranganathan S: SPdb-a signal peptide database. BMC Bioinforma 2005, 6(1):249.
44. Dallal Bashi Z, Hegedus DD, Buchwaldt L, Rimmer SR, Borhan MH: Expression and regulation of Sclerotinia sclerotiorum necrosis and ethylene-inducing peptides (NEPs). Mol Plant Pathol 2010, 11(1):43-53.

45. Kleemann J, Rincon-Rivera $\amalg$, Takahara H, Neumann U, van Themaat EVL, van der Does HC, Hacquard S, Stüber K, Will I, Schmalenbach W: Sequential delivery of host-induced virulence effectors by appressoria and intracellular hyphae of the phytopathogen Colletotrichum higginsianum. PLoS Pathog 2012, 8(4):e1002643.

46. Matei E, Louis JM, Jee J, Gronenborn AM: NMR solution structure of a cyanovirin homolog from wheat head blight fungus. Proteins Structure Function Bioinformatics 2011, 79(5):1538-1549.

47. Seidl V, Marchetti M, Schandl R, Allmaier G, Kubicek CP: Epl1, the major secreted protein of Hypocrea atroviridis on glucose, is a member of a strongly conserved protein family comprising plant defense response elicitors. FEBS J 2006, 273(18):4346-4359.

48. Jeong JS, Mitchell TK, Dean RA: The Magnaporthe grisea snodprot1 homolog, MSP1, is required for virulence. FEMS Microbiol Lett 2007 273(2):157-165.

49. Song J, Win J, Tian M, Schornack S, Kaschani F, llyas M, van der Hoorn RAL, Kamoun S: Apoplastic effectors secreted by two unrelated eukaryotic plant pathogens target the tomato defense protease Rcr3. Proc Natl Acad Sci 2009, 106(5):1654

50. McCann HC, Nahal H, Thakur S, Guttman DS: Identification of innate immunity elicitors using molecular signatures of natural selection. Proc Natl Acad Sci 2012, 109(11):4215-4220.

51. Pedersen C, van Themaat EVL, McGuffin LJ, Abbott JC, Burgis TA, Barton G, Bindschedler LV, Lu X, Maekawa T, Weßling R, Cramer R, Thordal-Christensen $H$, Panstruga $R$, Spanu PD: Structure and evolution of barley powdery mildew effector candidates. BMC Genomics 2012, 13(1):694

52. Sella L, Gazzetti K, Faoro F, Odorizzi S, D'Ovidio R, Schäfer W, Favaron F: A Fusarium graminearum xylanase expressed during wheat infection is a necrotizing factor but is not essential for virulence. Plant Physiol Biochem 2013, 64:1-10

53. Sansen S, De Ranter CJ, Gebruers K, Brijs K, Courtin CM, Delcour JA, Rabijns A: Structural basis for inhibition of Aspergillus niger xylanase by Triticum aestivum xylanase inhibitor-I. J Biol Chem 2004, 279(34):36022-36028.

54. Yoshizawa T, Shimizu T, Hirano $H$, Sato $M$, Hashimoto $H$ : Structural basis for inhibition of xyloglucan-specific endo- $\beta$-1, 4-glucanase (XEG) by XEG-protein inhibitor. J Biol Chem 2012, 287(22):18710-18716.

55. van der Does HC, Rep M: Virulence genes and the evolution of host specificity in plant-pathogenic fungi. Mol Plant Microbe Interact 2007, 20(10):1175-1182.

56. Feil SC, Lawrence S, Mulhern TD, Holien JK, Hotze EM, Farrand S, Tweten RK, Parker MW: Structure of the lectin regulatory domain of the cholesteroldependent cytolysin lectinolysin reveals the basis for its lewis antigen specificity. Structure 2012, 20(2):248-258.

57. Willis C, Wang CK, Osman A, Simon A, Pickering D, Mulvenna J, RiboldiTunicliffe A, Jones MK, Loukas A, Hofmann A: Insights into the membrane interactions of the saposin-like proteins Na-SLP-1 and AC-SLP-1 from human and dog hookworm. PLoS One 2011, 6(10):e25369.

58. Liberman N, Dym O, Unger T, Albeck S, Peleg $Y$, Jacobovitch $Y$, Branzburg A Eisenstein M, Marash L, Kimchi A: The crystal structure of the C-terminal DAP5/p97 domain sheds light on the molecular basis for its processing by caspase cleavage. J Mol Biol 2008, 383(3):539-548.

59. Lee WS, Rudd JJ, Hammond Kosack KE, Kanyuka K: Mycosphaerella graminicola LysM effector-mediated stealth pathogenesis subverts recognition through both CERK 1 and CEBiP homologues in wheat. Mol Plant-Microbe Interact 2013, 27(3):236-243.

60. Brown NA, Antoniw J, Hammond-Kosack KE: The predicted secretome of the plant pathogenic fungus Fusarium graminearum: a refined comparative analysis. PLoS One 2012, 7(4):e33731.

61. Hegedus DD, Rimmer SR: Sclerotinia sclerotiorum: When "to be or not to be" a pathogen? FEMS Microbiol Lett 2005, 251(2):177-184.

62. Pollet A, Sansen S, Raedschelders G, Gebruers K, Rabijns A, Delcour JA Courtin CM: Identification of structural determinants for inhibition strength and specificity of wheat xylanase inhibitors TAXI-IA and TAXI-IIA. FEBS J 2009, 276(14):3916-3927.

63. Di Matteo A, Giovane A, Raiola A, Camardella L, Bonivento D, De Lorenzo G, Cervone F, Bellincampi D, Tsernoglou D: Structural basis for the interaction between pectin methylesterase and a specific inhibitor protein. Plant Cell Online 2005, 17(3):849-858. 
64. Hacquard S, Kracher B, Maekawa T, Vernaldi S, Schulze-Lefert P, van Themaat EVL: Mosaic genome structure of the barley powdery mildew pathogen and conservation of transcriptional programs in divergent hosts. Proc Natl Acad Sci 2013, 110(24):E2219-E2228.

65. Lahrmann U, Ding Y, Banhara A, Rath M, Hajirezaei MR, Döhlemann S, von Wirén N, Parniske M, Zuccaro A: Host-related metabolic cues affect colonization strategies of a root endophyte. Proc Natl Acad Sci 2013, 110(34):13965-13970.

66. Nielsen $\mathrm{H}$, Krogh A: Prediction of signal peptides and signal anchors by a hidden Markov model, Ismb. 1998:122-130.

67. Petersen TN, Brunak S, von Heijne G, Nielsen H: SignalP 4.0: discriminating signal peptides from transmembrane regions. Nat Methods 2011, 8(10):785-786.

68. Sonnhammer EL, von Heijne G, Krogh A: A hidden Markov model for predicting transmembrane helices in protein sequences. proc Int Conf Intell Syst Mol Biol 1998, 6:175-182.

69. Fankhauser N, Mäser P: Identification of GPI anchor attachment signals by a Kohonen self-organizing map. Bioinformatics 2005, 21(9):1846-1852.

70. Conesa A, Gotz S, Garcia-Gomez JM, Terol J, Talon M, Robles M: Blast2GO: a universal tool for annotation, visualization and analysis in functional genomics research. Bioinformatics 2005, 21(18):3674-3676.

71. Punta M, Coggill PC, Eberhardt RY, Mistry J, Tate J, Boursnell C, Pang N, Forslund K, Ceric G, Clements J, Heger A, Holm L, Sonnhammer EL, Eddy SR, Bateman A, Finn RD: The Pfam protein families database. Nucleic Acids Res 2012, 40(D1):D290-D301

72. Ba ANN, Pogoutse A, Provart N, Moses AM: NLStradamus: a simple Hidden Markov Model for nuclear localization signal prediction. BMC Bioinforma 2009, 10(1):202.

73. Östlund G, Schmitt T, Forslund K, Köstler T, Messina DN, Roopra S, Frings O, Sonnhammer EL: InParanoid 7: new algorithms and tools for eukaryotic orthology analysis. Nucleic Acids Res 2010, 38(suppl 1):D196-D203.

74. Wang D, Zhang Y, Zhang Z, Zhu J, Yu J: KaKs_Calculator 2.0: a toolkit incorporating gamma-series methods and sliding window strategies. Genomics, Proteomics Bioinformatics 2010, 8(1):77-80.

75. Stern A, Doron-Faigenboim A, Erez E, Martz E, Bacharach E, Pupko T: Selecton 2007: advanced models for detecting positive and purifying selection using a Bayesian inference approach. Nucleic Acids Res 2007, 35(suppl 2):W506-W511.

76. Zhang Y: I-TASSER server for protein 3D structure prediction. BMC Bioinforma 2008, 9(1):40

77. Pettersen EF, Goddard TD, Huang CC, Couch GS, Greenblatt DM, Meng EC, Ferrin TE: UCSF Chimera-a visualization system for exploratory research and analysis. J Comput Chem 2004, 25(13):1605-1612.

78. Grigoriev IV, Nikitin R, Haridas S, Kuo A, Ohm R, Otillar R, Riley R, Salamov A, Zhao X, Korzeniewski F, Smirnova T, Nordberg H, Dubchak IL, Shabalov I: MycoCosm portal: gearing up for 1000 fungal genomes. Nucleic Acids Res 2014, 42(D1):D699-D704

79. Felsenstein J: PHYLIP - Phylogeny Inference Package (Version 3.2). Cladistics 1989, 5:16-166.

80. Enright AJ, Van Dongen S, Ouzounis CA: An efficient algorithm for large-scale detection of protein families. Nucleic Acids Res 2002, 30(7):1575-1584.

81. Saunders DGO, Win J, Kamoun S, Raffaele S: Two-Dimensional data binning for the analysis of genome architecture in filamentous plant pathogens and other eukaryote. In Methods in Molecular Biology, Volume 1127. Seconth edition. Edited by Birch PRJ, Jones JT, Bos JIB. New Yor: Springer Science + Business Media; 2014

doi:10.1186/1471-2164-15-336

Cite this article as: Guyon et al:: Secretome analysis reveals effector candidates associated with broad host range necrotrophy in the fungal plant pathogen Sclerotinia sclerotiorum. BMC Genomics 2014 15:336.

\section{Submit your next manuscript to BioMed Central and take full advantage of:}

- Convenient online submission

- Thorough peer review

- No space constraints or color figure charges

- Immediate publication on acceptance

- Inclusion in PubMed, CAS, Scopus and Google Scholar

- Research which is freely available for redistribution

Submit your manuscript at www.biomedcentral.com/submit
Biomed Central 